\title{
ENDF/B-VIII.0 Covariance Data Development and Testing for Advanced Reactors
}

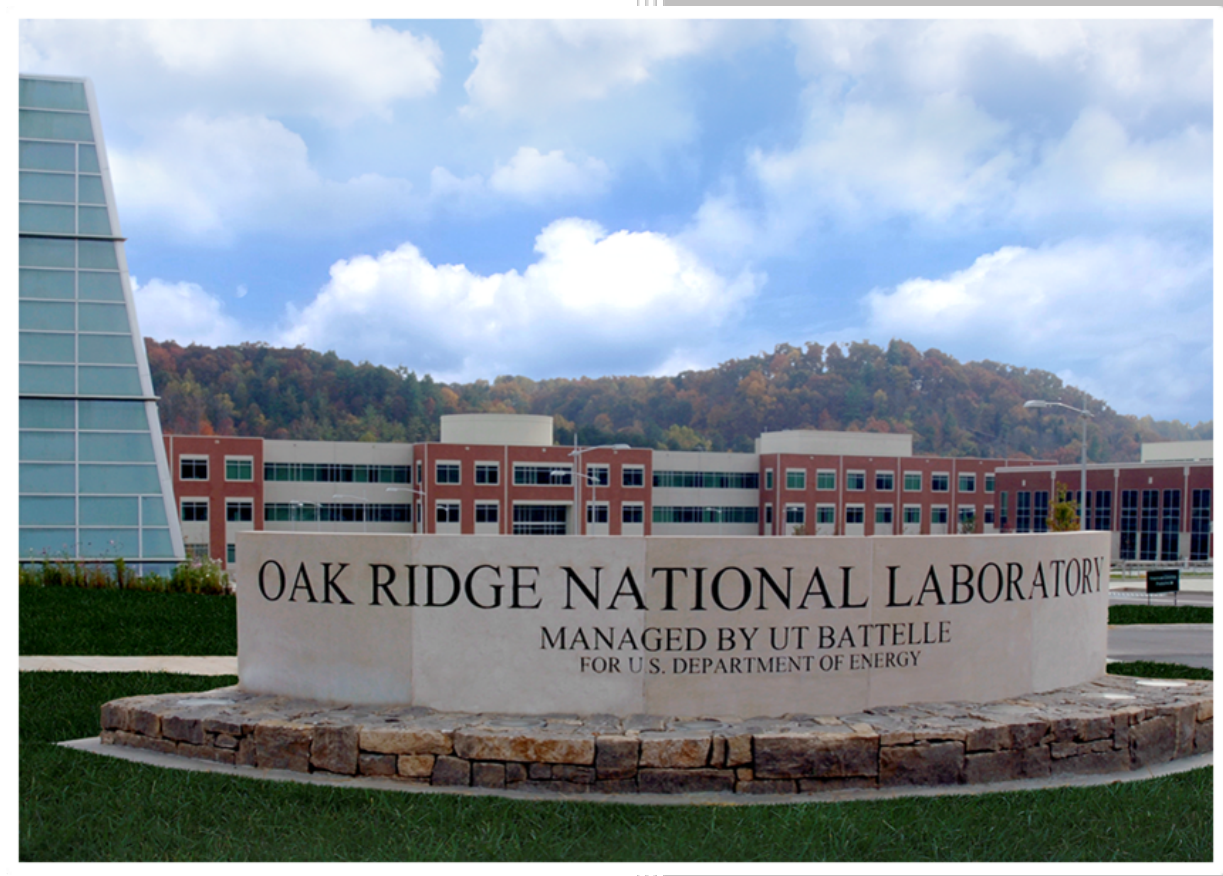

Approved for public release. Distribution is unlimited.
Vladimir Sobes

William J. Marshall

Doro Wiarda

Friederike Bostelmann

Andrew Holcomb

Bradley T. Rearden

March 2019 


\title{
DOCUMENT AVAILABILITY
}

Reports produced after January 1, 1996, are generally available free via US Department of Energy (DOE) SciTech Connect.

Website www.osti.gov

Reports produced before January 1, 1996, may be purchased by members of the public from the following source:

\author{
National Technical Information Service \\ 5285 Port Royal Road \\ Springfield, VA 22161 \\ Telephone 703-605-6000 (1-800-553-6847) \\ TDD 703-487-4639 \\ Fax 703-605-6900 \\ E-mail info@ntis.gov \\ Website http://classic.ntis.gov/
}

Reports are available to DOE employees, DOE contractors, Energy Technology Data Exchange representatives, and International Nuclear Information System representatives from the following source:

Office of Scientific and Technical Information

PO Box 62

Oak Ridge, TN 37831

Telephone 865-576-8401

Fax 865-576-5728

E-mail reports@osti.gov

Website http://www.osti.gov/contact.html

This report was prepared as an account of work sponsored by an agency of the United States Government. Neither the United States Government nor any agency thereof, nor any of their employees, makes any warranty, express or implied, or assumes any legal liability or responsibility for the accuracy, completeness, or usefulness of any information, apparatus, product, or process disclosed, or represents that its use would not infringe privately owned rights. Reference herein to any specific commercial product, process, or service by trade name, trademark, manufacturer, or otherwise, does not necessarily constitute or imply its endorsement, recommendation, or favoring by the United States Government or any agency thereof. The views and opinions of authors expressed herein do not necessarily state or reflect those of the United States Government or any agency thereof. 
Reactor and Nuclear Systems Division

\title{
ENDF/B-VIII.0 COVARIANCE DATA DEVELOPMENT AND TESTING FOR ADVANCED REACTORS
}

\author{
Vladimir Sobes \\ William J. Marshall \\ Fiederike Bostelmann \\ Andrew M. Holcomb \\ Bradley T. Rearden
}

Date Published: March 2019

Prepared by

OAK RIDGE NATIONAL LABORATORY

Oak Ridge, TN 37831-6283

managed by

UT-BATTELLE, LLC

for the

US DEPARTMENT OF ENERGY

under contract DE-AC05-00OR22725 



\section{CONTENTS}

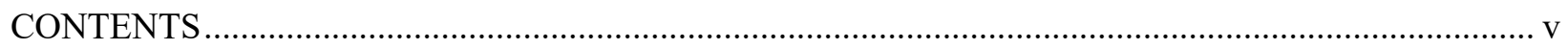

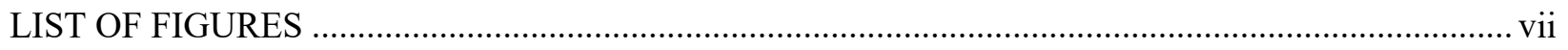

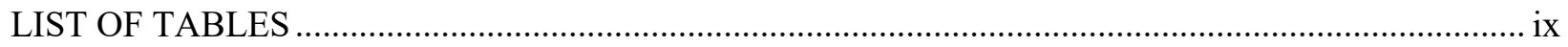

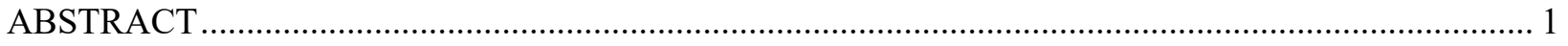

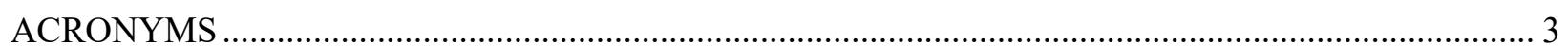

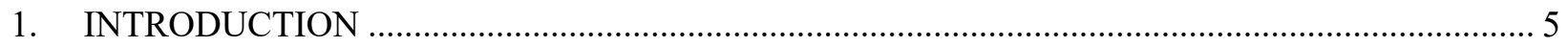

$1.1 \quad$ NUCLEAR DATA NEEDS FOR ADVANCED REACTORS ……..................................... 5

1.2 NUCLEAR DATA AND COVARIANCE ASSESSMENT …........................................... 7

ENDF/B-VIII.0 COVARIANCE DATA DEVELOPMENT AND TESTING.......................... 7

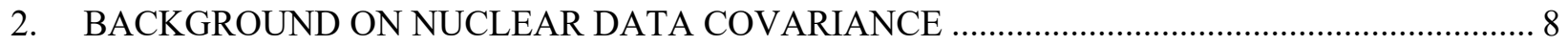

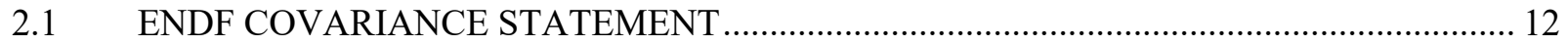

3. ENDF/B-VIII.0 Covariance Testing WITH INTEGRAL BENCHMARK EXPERIMENTS ........... 13

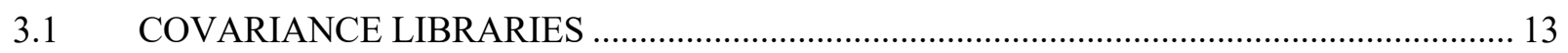

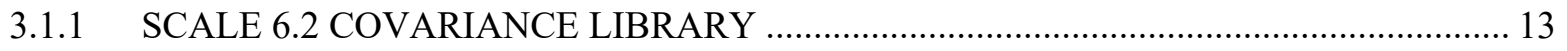

3.1.2 ENDF/B-VIII.0 EXPERIMENTAL COVARIANCE LIBRARY …................................. 13

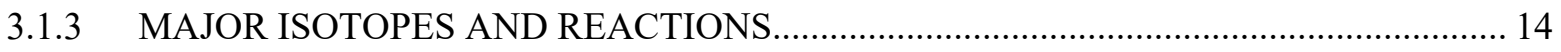

3.2 NUCLEAR DATA-INDUCED UNCERTAINTY IN VALID MODEL $K_{\text {EFF }}$ VALUES ......... 16

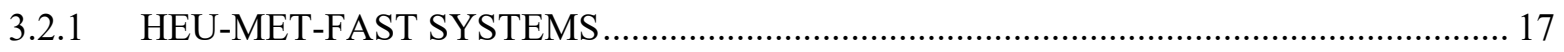

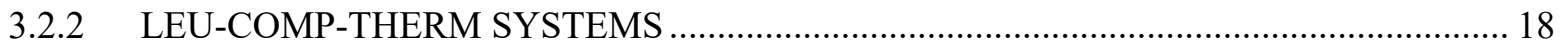

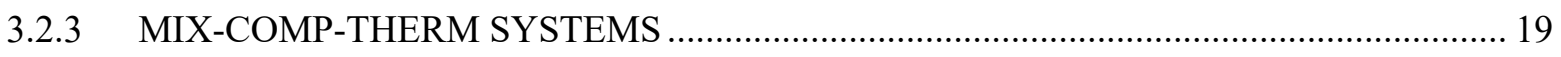

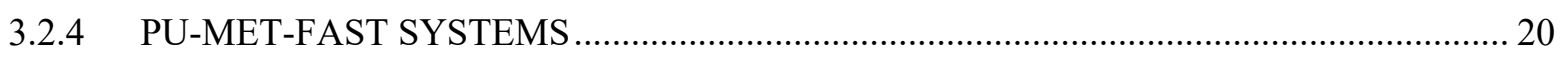

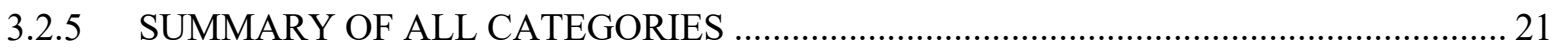

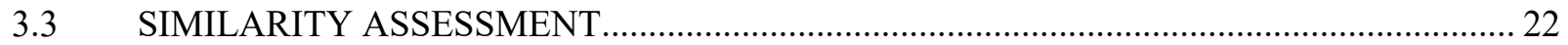

4. ENDF/B-VIII.0 Covariance Assessment For Advanced Reactor Applications ............................... 24

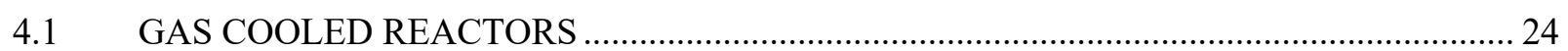

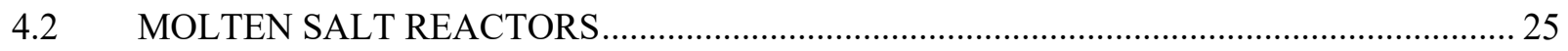

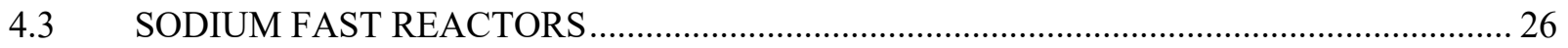

5. AUGMENTED ENDF/B-VIII.0 Covariance Development .......................................................... 26

5.1 THE APPROACH TO A FIRST AUGMENTED COVARIANCE MATRIX........................ 26

5.2 THE RESULTS OF AN AUGMENTED COVARIANCE MATRIX ..................................... 27

6. AUGMENTED ENDF/B-VIII.0 Covariance matrix results for integral experiments ...................... 33

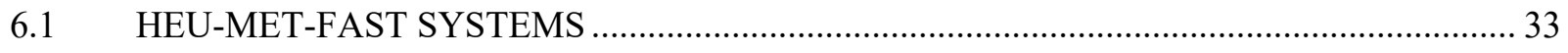

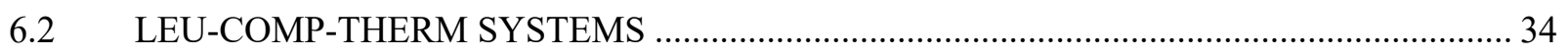

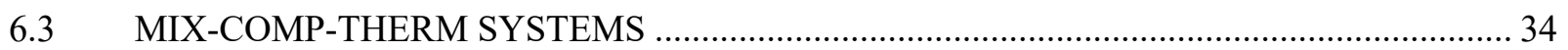

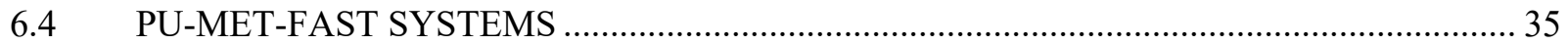




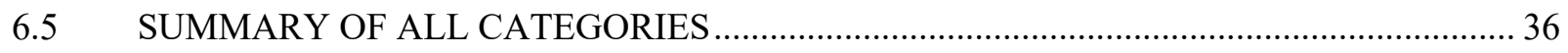

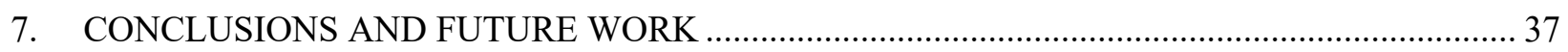

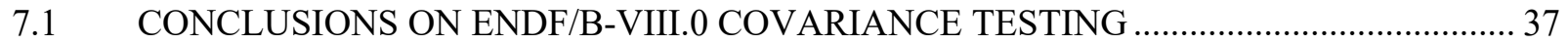

7.2 FOLLOW-ON WORK ON THE AUGMENTED COVARIANCE MATRIX ...................... 37

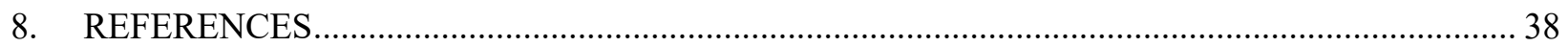





\section{LIST OF FIGURES}

Figure 1. Calculated flux spectra for several reactor concepts.

Figure 2. Neutron capture cross section of ${ }^{235} \mathrm{U}$ (left axis) and the relative uncertainty (right axis).......... 6

Figure 3. An experimental set up for neutron capture cross section measurements. ................................... 9

Figure 4. Total neutron cross section for ${ }^{65} \mathrm{Cu}$ (experimental data are the same in both panels and were

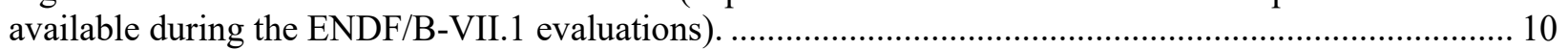

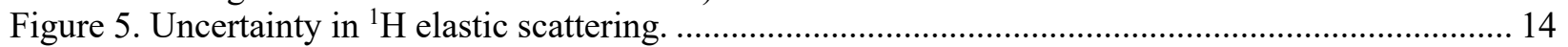

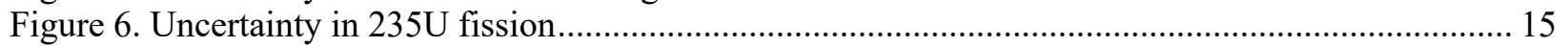

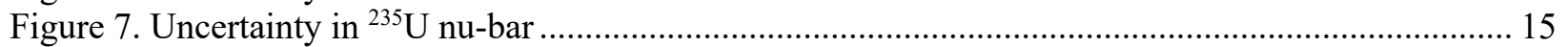

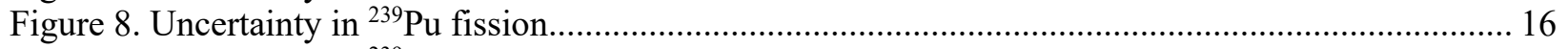

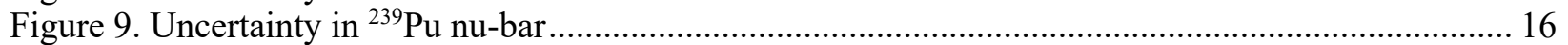

Figure 10. Results for HEU-MET-FAST systems. The experiments are sorted by their ICBSEP-assigned

number on the horizontal axis. C/E values are shown on the vertical axis.......................................... 17

Figure 11. Results for LEU-COMP-THERM systems. The experiments are sorted by their ICBSEP-

assigned number on the horizontal axis. C/E values are shown on the vertical axis............................... 19

Figure 12. Uncertainty in ${ }^{238} \mathrm{U}$ inelastic scattering ..................................................................... 19

Figure 13. Results for the MIX-COMP-THERM systems. The experiments are sorted by their ICBSEP-

assigned number on the horizontal axis. C/E values are shown on the vertical axis............................. 20

Figure 14. Results for PU-MET-FAST systems. The experiments are sorted by their ICBSEP-assigned

number on the horizontal axis. C/E values are shown on the vertical axis........................................... 21

Figure 15. Applicability of various critical experiments for validation of PWR spent fuel storage system

using SCALE 6.2 and ENDF/B-VIII.0 covariance libraries.............................................................. 23

Figure 16. Applicability of various critical experiments for validation of PWR spent fuel storage system

using SCALE 6.1 and ENDF/B-VIII.0 covariance libraries.................................................................. 24

Figure 17. Results for HEU-MET-FAST systems. The experiments are sorted by their ICBSEP-assigned

number on the horizontal axis. C/E values are shown on the vertical axis............................................. 33

Figure 18. Results for LEU-COMP-THERM systems. The experiments are sorted by their ICBSEP-

assigned number on the horizontal axis. C/E values are shown on the vertical axis.............................. 34

Figure 19. Results for the MIX-COMP-THERM systems. The experiments are sorted by their ICBSEP-

assigned number on the horizontal axis. C/E values are shown on the vertical axis............................. 35

Figure 20. Results for PU-MET-FAST systems. The experiments are sorted by their ICBSEP-assigned

number on the horizontal axis. $\mathrm{C} / \mathrm{E}$ values are shown on the vertical axis............................................ 36 



\section{LIST OF TABLES}

Table 1. Uncertainty Summary for Ten Categories of Experiments in VALID (Uncertainties are reported

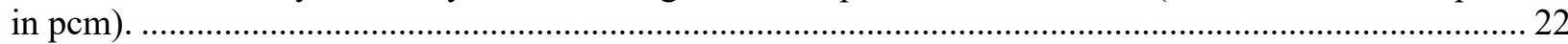

Table 2. Coarse group cross correlation estimates for ${ }^{239} \mathrm{Pu}$ based on including different VALID benchmarks in the TSURFER GLLS adjustment (in percent)........................................................... 28

Table 3. Coarse group cross correlation estimates for ${ }^{235} \mathrm{U}$ based on including different VALID benchmarks in the TSURFER GLLS adjustment (in percent)............................................................. 30 Table 4. Coarse group cross correlation estimates for ${ }^{238} \mathrm{U}$ fast fission to fast nu-bar based on including different VALID benchmarks in the TSURFER GLLS adjustment (in percent). .................................. 31 Table 5. Estimations of the coarse group cross-correlations for the first augmented ENDF/B-VIII.0 library

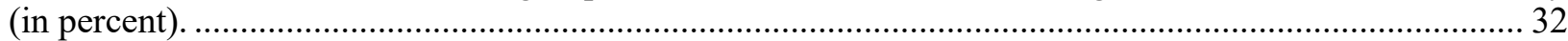
Table 6. Uncertainty Summary for Ten Categories of Experiments in VALID (Uncertainties are reported

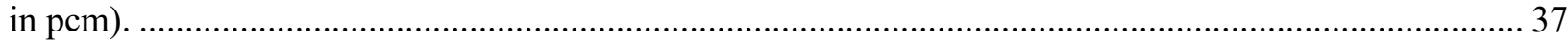





\begin{abstract}
This report documents the ENDF/B-VIII.0 covariance data development and testing performed at Oak Ridge National Laboratory performed under the auspices of the US Department of Energy Office of Nuclear Energy. This work supports modeling and simulation of advanced reactor designs in the US by analyzing, validating and improving the nuclear data that provide underlying physics for calculations. This report assesses nuclear data uncertainty information for neutron cross sections, provided in the form of covariance matrices, in the 2018 ENDF/B-VIII.0 library. A review and interpretation of some of the issues with the current covariance library is provided. A new approach to augment the ENDF/B-VIII.0 covariance matrix to accurately reflect uncertainties in the results of modeling and simulation reliant on the current nuclear data library is documented. Rigorous testing of the ENDF/B-VIII.0 covariance data is documented. Finally, the performance of the ENDF/B-VIII.0 covariance data for advanced reactor applications is assessed.
\end{abstract}




\section{ACRONYMS}

\begin{tabular}{|c|c|}
\hline $\mathrm{C} / \mathrm{E}$ & calculated-to-experiment \\
\hline CSEWG & Cross Section Evaluation Working Group \\
\hline DoD & US Department of Defense \\
\hline DOE & US Department of Energy \\
\hline DOE-NE & DOE Office of Nuclear Energy \\
\hline DOE-SC & DOE Office of Science \\
\hline EALF & energy of the average lethargy causing fission \\
\hline ENDF & Evaluated Nuclear Data Format \\
\hline GLLS & generalized linear least squares \\
\hline GRS & Global Research for Safety \\
\hline HEU & highly enriched uranium \\
\hline HMF & highly enriched uranium metal fast spectrum \\
\hline HST & highly enriched solution thermal spectrum \\
\hline HTR & high-temperature gas-cooled reactor \\
\hline HTTR & high-temperature engineering test reactor \\
\hline ICSBEP & International Criticality Safety Benchmark Evaluation Project \\
\hline IMF & intermediate-enriched uranium metal fast spectrum \\
\hline IRPhEP & The International Reactor Physics Experiment Evaluation Project \\
\hline LCT & low-enriched uranium compound thermal spectrum \\
\hline LEU & low enriched uranium \\
\hline LST & low-enriched solution thermal spectrum \\
\hline LWR & light water reactor \\
\hline MSF & mixed uranium, and plutonium solution fast spectrum \\
\hline $\mathrm{MCT}$ & mixed uranium, and plutonium oxide compound thermal spectrum \\
\hline MST & mixed uranium, and plutonium solution thermal spectrum \\
\hline MSR & molten salt reactor \\
\hline NA-22 & Office of Defense Nuclear Nonproliferation \\
\hline NCSP & Nuclear Criticality Safety Program \\
\hline NEA & Nuclear Energy Agency \\
\hline NEET & Nuclear Energy Enabling Technology \\
\hline NEUP & Nuclear Energy University Program \\
\hline NNSA & National Nuclear Security Administration \\
\hline $\mathrm{NRC}$ & US Nuclear Regulatory Commission \\
\hline OECD & Organization for Economic Co-operation and Development \\
\hline ORNL & Oak Ridge National Laboratory \\
\hline PMF & plutonium metal fast spectrum \\
\hline PST & plutonium solution thermal spectrum \\
\hline PWR & pressurized water reactor \\
\hline SFR & sodium-cooled fast reactor \\
\hline VALID & Verified, Archived Library of Inputs and Data \\
\hline XSUSA & Cross Section Uncertainty and Sensitivity Analysis \\
\hline
\end{tabular}




\section{INTRODUCTION}

\subsection{NUCLEAR DATA NEEDS FOR ADVANCED REACTORS}

Unlike light water reactors (LWRs), most advanced reactor concepts do not benefit from decades of operational experience and supporting infrastructure that lead to increased confidence in predictive results from modeling and simulation. Many advanced reactor concepts, including high-temperature gas reactors (HTGRs), fluoride salt-cooled high temperature reactors (FHRs), liquid fueled molten salt reactors (MSRs), sodium fast reactors (SFRs), and microreactors, implement unique materials and neutron spectra that are unique from LWRs. Advanced reactors push the envelope of very high burnup and high assay Low Enriched Uranium (LEU) fuel. Further nontraditional fuel forms and general lack of experienced analysts who understand design and safety aspects of these systems all present challenges for the advanced reactor community.

The current economic and regulatory environment does not allow rapid prototyping and construction of test and demonstration nuclear power plants for the proposed advanced reactors that was available for the LWR concepts at the dawn of the nuclear-power-age. Therefore, the advanced reactor concepts must now rely much more on advanced modeling and simulation tools to understand and predict the behavior of their reactor design. The accuracy of the predictions of modern modeling and simulation software, in particular for the neutronics analysis of nuclear reactors, is almost entirely dependent on the quality of the physics parameters implemented in the simulation. For the case of neutronics analysis, this input data is the fundamental nuclear data. Due to differences in neutron spectra and materials, many advanced reactor concepts rely on different aspects of nuclear data than traditional LWRs. Figure 1, presents a comparison of the representative flux profiles for a traditional LWR versus several advanced reactor concepts. Figure 2 , presents the neutron capture cross section for ${ }^{235} \mathrm{U}$ and the associated evaluated uncertainty referenced to the right axis.

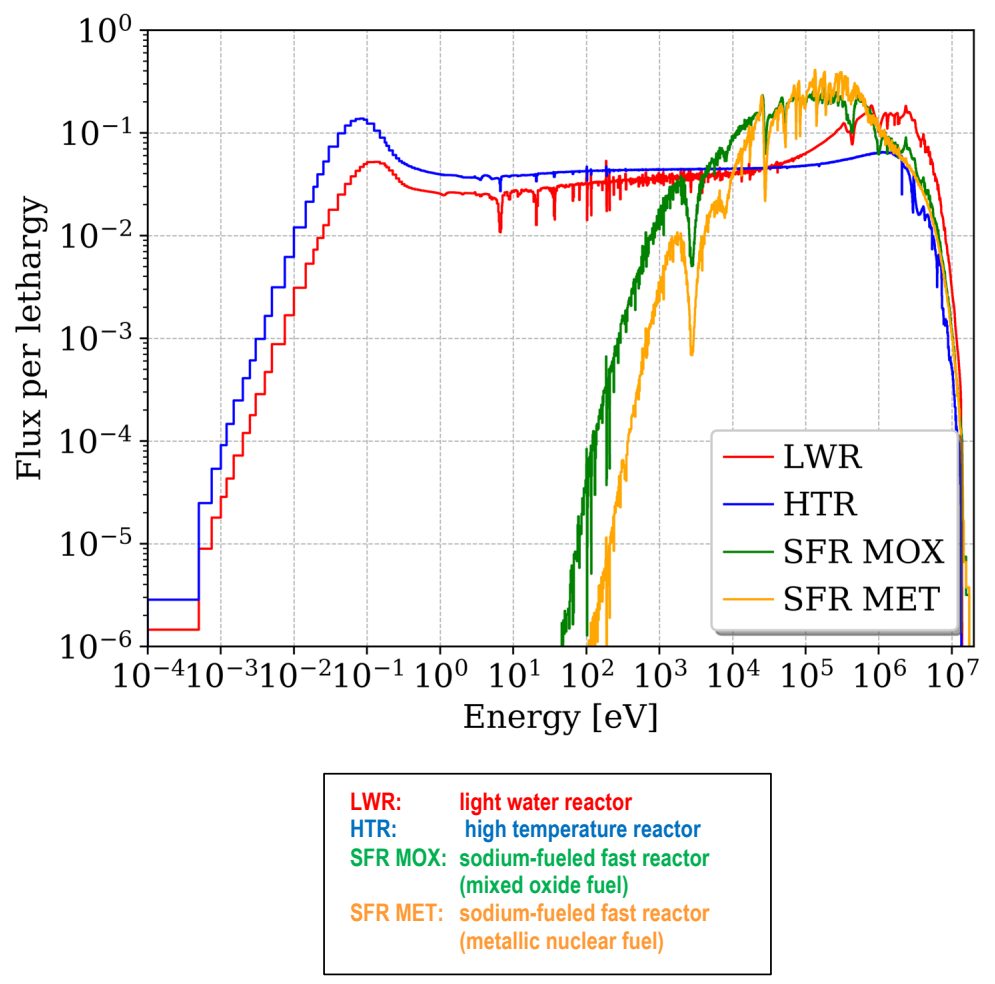

Figure 1. Calculated flux spectra for several reactor concepts. 


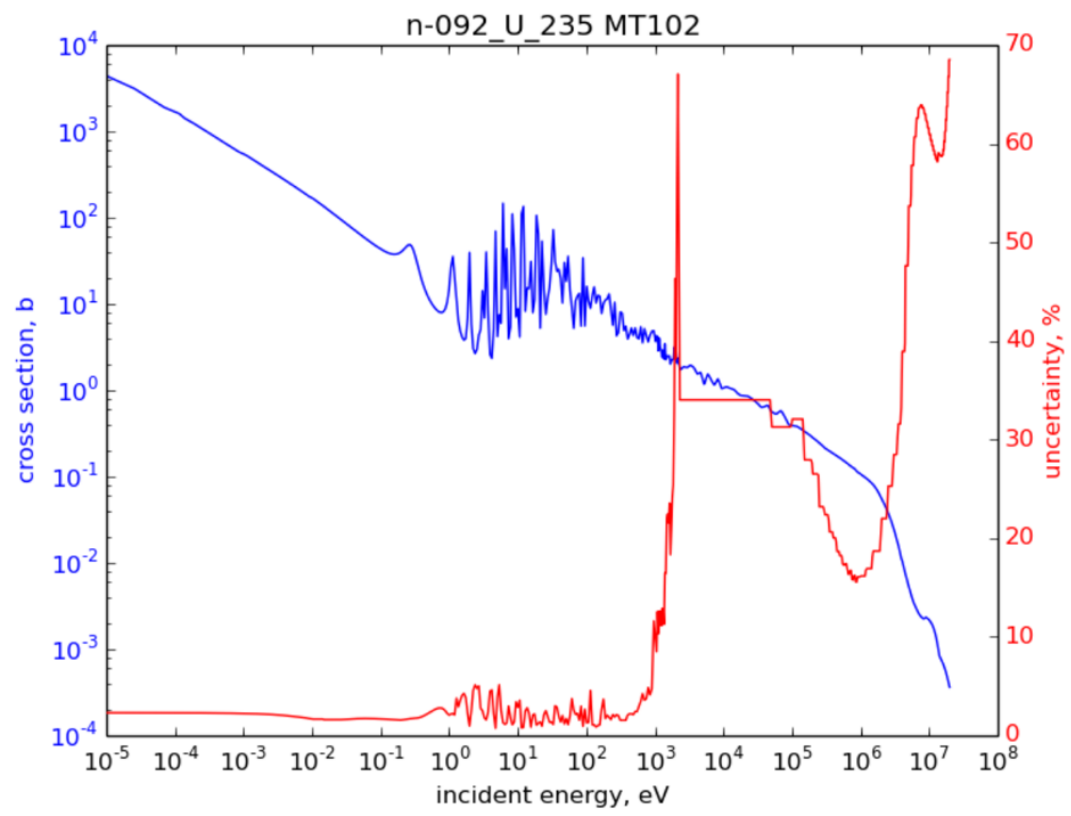

Figure 2. Neutron capture cross section of ${ }^{235} \mathbf{U}$ (left axis) and the relative uncertainty (right axis).

Notice that while the uncertainty on the capture cross section of ${ }^{235} \mathrm{U}$ is small, on the order of $5 \%$, in the energy region corresponding the peak in the LWR flux spectrum, the uncertainty spikes up to above $60 \%$ in the energy regions corresponding the calculate flux spectra for the SFR designs. This example demonstrates that even through nuclear data are well known for the important aspects for one reactor concept, that same nuclear data may not be well known in the important aspects of another reactor concept, even for perhaps the best studied nucleus of all, ${ }^{235} \mathrm{U}$.

In the recent decades nuclear data development has been supported by specific programs such as the US DOE Office of Nuclear Physics (DOE-NP), US DOE Nuclear Criticality Safety Program (NCSP), National Nuclear Security Administration's Defense Nuclear Non-proliferation Program (NA-22), US DOE Office of Naval Reactors (DOE-NR), Defense Treat Reduction Agency (DTRA), as well as international organizations such as the International Atomic Energy Agency (IAEA). Activities supported by these programs have produced new differential physics experiments, data processing and comparison to and optimization with applications in their interest. One of the key issues is that when nuclear data research, seemingly a fundamental physical pursuit, is funded by specific programs with particular application interests, the evaluation of the updated nuclear data is biased to subjective to best meet the application needs of the sponsoring organization. Therefore, if the updated nuclear data benefit, or do not disrupt, applications of interest to these agencies, the new evaluation is approved and accepted into the National Nuclear Data Center for distribution with the next revision of ENDF. Notably absent from the list of agencies that sponsor nuclear data activities are the US DOE Office of Nuclear Energy (DOE-NE) and the US Nuclear Regulatory Commission (NRC).

The Nuclear Data and Benchmarking program was proposed in 2018 as a new Nuclear Energy Enabling Technology Crosscutting Program (NEET) supported DOE-NE. The goal of the program is to partner with industry, the NRC and other programs to improve nuclear data and benchmarking of modeling and simulation for the advanced reactors in the US. This program aims to achieve this by, 1) Identifying the priority needs for nuclear data and benchmarking 2) performing new data measurements and evaluations 3) supporting integral experiments and handbooks and 4) participating in application benchmark studies.

In Fiscal Year 2018 (FY18) the Nuclear Data and Benchmarking Program was organized into 4 categories, 1) nuclear data and validation studies, 2) nuclear data generation, 3) international benchmarking activities 4) university projects. In the first of these categories, nuclear data and validation 
studies, a gap analysis of nuclear data was performed for nonLWR reactors and the validation basis, from the nuclear data perspective, for the transportation of high-assay LEU was investigated. The results of both of these validation studies are detailed in a sister-report accompanying this one.

In the second category, on the generation of new nuclear data, three tasks were performed. The first, was investigation and generation of application driven covariance data, which is the topic of this report. The second was improvements of nuclear data for depletion, activation, and decay. Finally, new measurement was approved for the ${ }^{238} U(n, n$ ') reaction with the associated uncertainty estimated through competitive award issued under a Funding Opportunity Announcement issued under the auspices of the Nuclear Data Interagency Working Group (NDIWG), in collaboration with DOE-NP.

In the third category, the Nuclear Data and Benchmarking Program supported the international collaboration for Multi-Physics Experimental Data, Benchmark, and Validation as well as the International Physics Benchmark Programs: the International Criticality Safety Benchmark Evaluation Project (ICSBEP) and International Reactor Physics Experiment Evaluation Project (IRPhEP). Finally, the Nuclear Data and Benchmarking Program participated in the Nuclear Energy University Program (NEUP) funding opportunity announcement and is launching two NEUP University projects researching thermal scattering law development. Namely, the generation of thermal scattering data for graphite and the generation of thermal scattering sensitivity/uncertainty capabilities.

\subsection{NUCLEAR DATA AND COVARIANCE ASSESSMENT}

The objective of the nuclear data and covariance assessment studies is the identification and confirmation of priority nuclear data needs, especially supporting the Nuclear Energy Institute's Advanced Reactor Technical Working Groups in support of the NDIWG.

The 2018 ENDF/B-VIII.0 library contains significantly more covariance data than 2011 ENDF/B-VII.1 library. The new data include many materials of key interest to the advanced reactor community that will require investigation before confidently moving to production use. Additionally, the ENDF/B-VIII.0 data include a disclaimer that the covariances should not be applied in applications without first implementing a sensitivity/uncertainty-based data adjustment using relevant integral benchmarks. A first augmented covariance data set has been produced from ENDF/B-VIII.0 data, and its performance is assessed in this report using application systems relevant to the advanced reactor community.

Many updates in nuclear data have been implemented over the past several ENDF/B releases that produce significant changes in calculated values for advanced reactors. Additional gaps in nuclear data have been identified that have unknown effects on reactor calculations. Assessment of the performance of several nuclear data libraries has been performed using continuous-energy and multigroup analysis for systems relevant to the advanced reactor community. Where feasible, uncertainties in these systems due to nuclear data uncertainties are also assessed. The findings of this study are documented in a sister-report accompanying this one [1].

\subsection{ENDF/B-VIII.0 COVARIANCE DATA DEVELOPMENT AND TESTING}

This report documents the work performed at ORNL in FY18 on developing an augmented ENDF/BVIII.0 nuclear data covariance library and testing the ENDF/B-VIII.0 covariance data as well as the augmented library. The report, following, is laid out in seven sections. Section 2 will provide some background overview origins of nuclear data covariance and discuss some of the difficulties of covariance estimation. Section 2 will conclude with a section devoted to discussing the statement published by the Cross Section Evaluation Working Group (CSEWG) covariance committee with respect to the interpretation of the nuclear data covariance provided with the ENDF/B-VIII.0 library. Section 3 will present the findings of testing of the ENDF/B-VIII.0 covariance. Section 4 will follow with an assessment of the performance of the ENDF/B-VIII.0 covariance library for select advanced reactor applications. Section 5 summarizes the work carried out at ORNL to produce the first augmented 
ENDF/B-VIII.0 covariance library as suggested by the guidance of the CSEWG covariance committee. Section 6 will present a comparison of the performance of the ENDF/B-VIII.0 original covariance library and the augmented library. Lastly, the summary of the conclusions and future work will be outlined in Section 7.

\section{BACKGROUND ON NUCLEAR DATA COVARIANCE}

In order to understand the origins and nature of nuclear data uncertainty one must first understand the nuclear data pipeline; how nuclear data is measured, evaluated, processed, validated and used. Even before that, though, let us discuss a more fundamental question, "Can't we just calculate the neutron cross sections for fundamental physics?"

On the surface, it would appear that one should be able to calculate the neutron cross sections (here taken as a representative example of the general nuclear data) should be computable from fundamental physical principles. The world of nuclear interactions is governed by the laws of quantum mechanics and by the axioms of quantum physics, one is guaranteed that solution, the wavefunctions, of the Schrodinger equations contain all of the information necessary to completely describe the system. However, not only it is currently unfeasible to solve a 236-body quantum mechanical problem (1 neutron +235 nucleons of ${ }^{235} \mathrm{U}$ ) we do not even know what equation to solve. That is because the nuclear potential, the strong nuclear force which governs nucleon-nucleon interactions, necessary for the solution of the Schrödinger equation is not well understood. The current knowledge of the strong nuclear force does allow for some limited predictive power in the calculation of nuclear cross sections averaged over wide-ranges of energy, however, the detailed behavior, especially in the resonance region is completely out of reach.

Due to the fact that nuclear cross sections cannot be calculated from first principles, we must conduct difficult and detailed experiments to measure the cross sections experimentally. The highest quality measurements, with experimental uncertainties less than 5\%, are incredibly complicated and the machines necessary for some experiments measure on the order of hundreds of meters. Figure 3 presents a photograph of a neutron capture measurement setup to give a perspective of the complicated set up. Given the complexity of the experiments it is unsurprising that the measurements are reported with an estimated uncertainty. The sources of uncertainty in a cross section measurement experiment can include uncertainties associated with background subtraction and normalization of data, detector counting statistics and detector efficiency as well as fundamental energy resolution of the machine. 


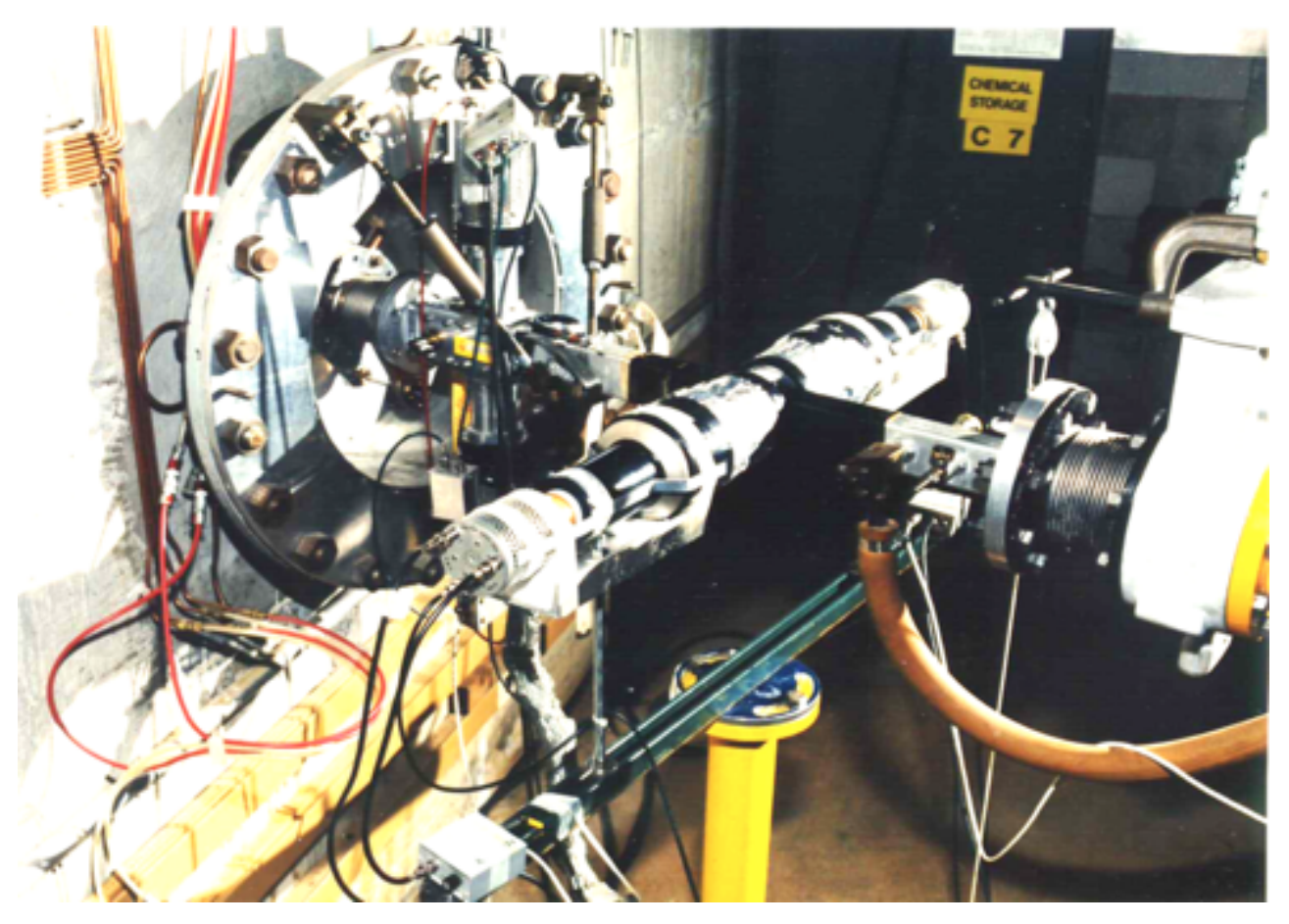

Figure 3. An experimental set up for neutron capture cross section measurements.

As the next step in the nuclear data pipeline, an evaluator, preferably unconnected to the experimentalists, will collect experimental measurements of the same or related nuclear data quantities from several different experimental facilities from around the world and evaluate them holistically in combination is historically archived experimental data. This is an import step in the nuclear data pipeline, as the evaluator is tasked with combining the parametric quantum models of nuclear data with the experimental results to produce a single mean value for the nuclear data quantity and the estimate of the uncertainty. One of the challenges that evaluators often face are discrepant measurements of the same quantity done at different facilities. This also serves as a demonstration for the necessity of the evaluation step; all of the experimental measurements are done on the same physical quantity which only has one value, but the results are not only uncertain, they can also be discrepant, and it is up to the evaluator to set a value and bound it by an uncertainty estimate. Lastly, a human element must also be considered when looking at the nuclear data pipeline. As Figure 4 demonstrates, due to different reasons, evaluations of experimental data can have downfalls as well. These downfalls can come from a variety of reasons, human errors, typos, lack of significance, etc. 


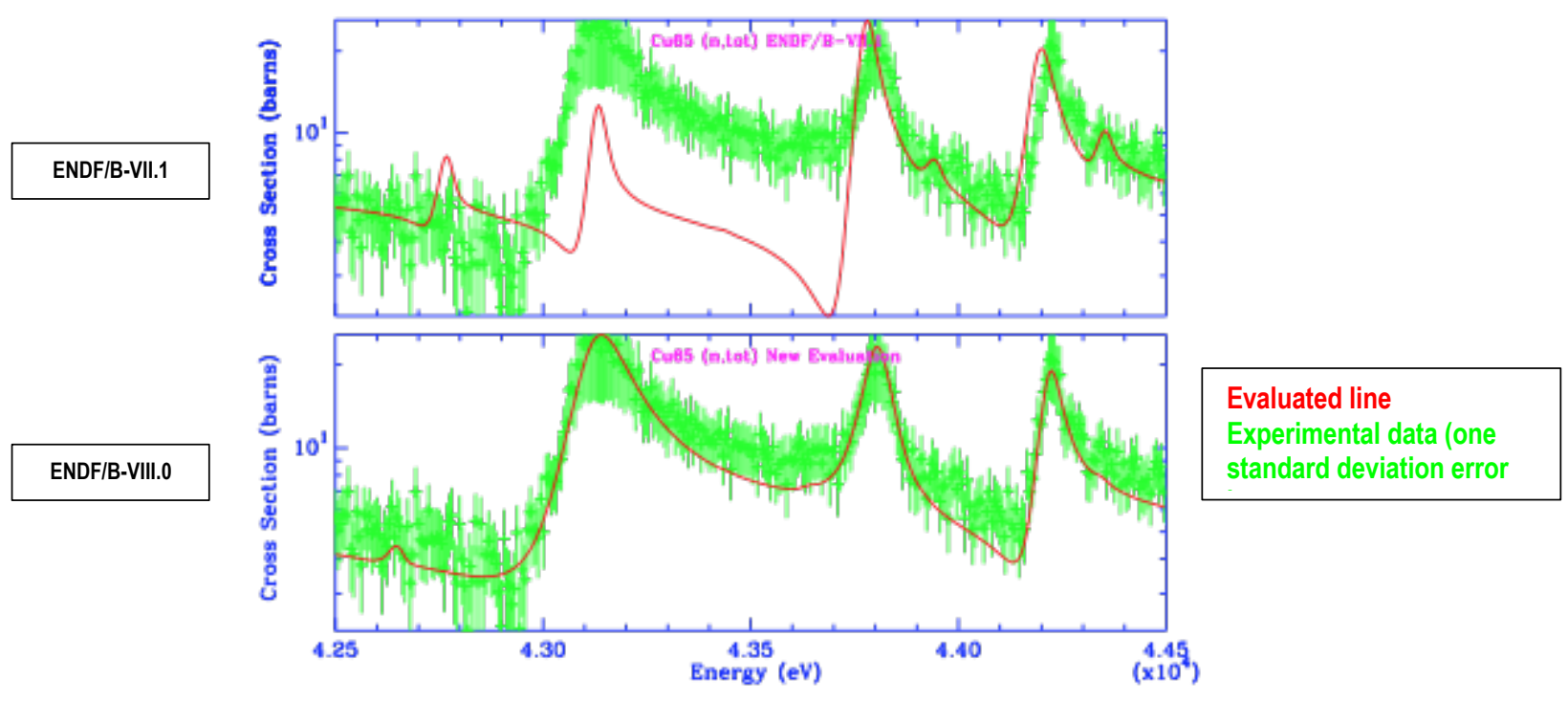

Figure 4. Total neutron cross section for ${ }^{65} \mathrm{Cu}$ (experimental data are the same in both panels and were available during the ENDF/B-VII.1 evaluations).

At this point, it is important to discuss what is the interpretation and ambiguity of nuclear data covariance. It is crucially important to understand that the covariance data reported in the ENDF/B libraries is not an estimation of a physical quantity. It is rather a statement of the belief or a confidence in the estimated mean value. The interpretation of the covariance data is similar of the Bayesian interpretation of statistics. That is, we believe that there is only one true value that exists in nature for each nuclear data quantity, e.g. the neutron capture cross section of ${ }^{235} \mathrm{U}$ at an incident neutron energy of $0.0253 \mathrm{eV}$. However, that value is unknown to us. Therefore, we report our best estimate of the true value with the mean value in the ENDF/B files and report an associated confidence interval, which can be calculated from the covariance data, that the true value lies within the confidence interval around the reported mean value. That is, the mean value in the ENDF file, estimates a true physical quantity, but the covariance is merely a statement of the degree of knowledge.

With this in mind, it is not unreasonable to have different covariance data with roughly similar mean value across the different international nuclear data projects. However, two points should draw closer scrutiny. First, if mean nuclear data values disagree between different nuclear data libraries outside the overlap of their confidence intervals. This is interpreted that we cannot agree on the value. The second point is if different nuclear data libraries have vastly different covariance estimates. This is interpreted as we cannot agree on how well do we know a particular quantity.

The next point is that uncertainty estimates, or associated confidence intervals, can be statistically argued to be either too small or too large. First, the statistical argument. The statistical interpretation of a confidence interval means that the more samples that are analyzed the stronger the conclusion. A simple example is if one experiment is inside of one standard deviation error bars, it is difficult to say whether the confidence interval is too large, however, if we find that out of 100 experiments all 100 are inside of one standard deviation error bars, then the argument to reduce the confidence intervals is much stronger. Next, using the statistical interpretation, it is easy to understand the argument that if too much of the data continue to fall within the uncertainty estimate, perhaps the uncertainty estimate is too large and viceversa.

There is one further ambiguity that must be considered in the interpretation of ENDF/B-VIII.0 covariance data. This is the ambiguity about the intention of the evaluator of reporting known-unknown versus unknown-unknowns and whether any estimations of the bias should be included and reported in a single covariance matrix. So far, there has not been a clear opinion from the nuclear data community on 
whether the reported nuclear data uncertainties should estimate the uncertainty on the mean values that can be calculated directly from the evaluation methods and reported experimental uncertainties (knownunknowns) or whether, the evaluator should make a best estimate of what uncertainties were not quantified and incorporate that into the covariance evaluation. The top panel of figure 4, provides an obvious example of where the estimate of the mean value of the cross section is likely to be many standard deviations off the true value if only a systematic evaluation of the uncertainty is reported.

Overall, it has been the opinion of the CSEWG community that the nuclear data covariance in the ENDF/B-VII.1 was overly optimistic. That is, the uncertainty estimates on the mean values of the nuclear data for many important reactions were on the border or below what can be measured by the current state of the art experimental methods. For this reason, many uncertainty estimates were increased in the library update from ENDF/B-VII.1 to ENDF/B-VIII.0. Some of these changes will be discussed in Section 3. Further, for some nuclides, the evaluators chose to systematically increase the uncertainty estimates to approximate the amount of unknown-unknowns.

After the evaluation of the differential experimental data is complete, in the nuclear data pipeline, the next step is testing of the evaluations on a set of integral experiments. As was discussed in Section 1, traditionally, the integral experiments used for validating nuclear data in the CSEWG community, have been a set of critical experiments from the ICSBEP. At this step, the results of the simulations of the integral experiments are compared to the experimentally measured values and the quality of the evaluation is judged by the discrepancy in the compared values.

It is important to note here, that the sensitivity of many of these integral systems to fundamental nuclear data can be quite large. A trivial example is that a $1 \%$ change in the average number of neutrons per fission (nu-bar) released by ${ }^{235} \mathrm{U}$ would change the k-effective of a sphere of high-enriched uranium by $1000 \mathrm{pcm}$. The integral benchmarks, especially with simple geometric configurations have much smaller experimental uncertainties that $1000 \mathrm{pcm}$. On the other hand, differential measurements of nu-bar are limited by uncertainties on the order of $0.5-1 \%$. This comparison gives the necessary space for feedback to occur between the evaluation and the validation part of the nuclear data pipeline. The evaluator has enough room, to make slight adjustments to the mean values that will result in improved performance of the evaluation in the calculation of the integral experiments, while, at the same time, be indistinguishable from the point of view of differential experimental measurements. However, this practice creates an inconsistency when considering the uncertainty data.

The process of adjusting the mean values within the ambiguity of the differential experimental data results in good agreement between the integral experiments and the calculations of their models. However, when this is compared against the calculated uncertainty on the simulations due to uncertainty in nuclear data generated from analysis of differential experiments it is often the case that the propagated uncertainty bands dwarf the observed discrepancies. This can be illustrated by inverting the earlier example of nu-bar. A realistic uncertainty estimates for a differential measurement of nu-bar on the order of $1 \%$ will alone propagate to an uncertainty of $1000 \mathrm{pcm}$ on the calculated value of the model of an integral experiment.

Having adjusted the mean values in such a way to achieve better agreement of the modeling predictions with the results of integral experiments does result in better predictive power of the modeling codes for integral systems which are similar to those used in the adjustment. From the point of view of the fundamental nuclear data, the knowledge that has been gained from the feedback loop is not as much in the determination of the individual nuclear data quantities but rather an increased understanding of the behavior of those quantities in particular combinations. To carry forward the example of nu-bar, a critical sphere of uranium metal does not say as much about the value of nu-bar as it says about the collective value of nu-bar multiplied by the fission cross section and divided by the absorption cross section. In the uncertainty representation, this increase in knowledge should be reflected in the cross-correlations between different reactions and different isotopes.

The good agreement between integral experiments and their modeling as will be discussed in Section 3, is a combination of having adjusted the mean values to get a better agreement for certain integral 
benchmarks and a true increase in knowledge of the different nuclear data quantities in particular combinations. Therefore, the uncertainty information provided with the nuclear data files should reflect the fact that for individual nuclear data quantities, knowledge is limited by the capabilities of state-of-the art differential experiments, but the knowledge of those quantities in particular combinations can be much better than predicted from their individual uncertainties.

Lastly, the problem of extrapolation, introduced in Section 1, must also be addressed. The covariance data must be such that it reflects our increased ability to predict certain integral quantities due to considering similar quantities in the feedback loop of the nuclear data pipeline. However, the covariance data must also reflect that integral quantities unrelated to those which were used in the adjustment are as uncertain as the individual nuclear data quantities are based on differential experimental measurements or more in the error which were previously compensating were to be additive.

\subsection{ENDF COVARIANCE STATEMENT}

The ENDF/B-VIII.0 library was published with the following interpretation of the covariance data.

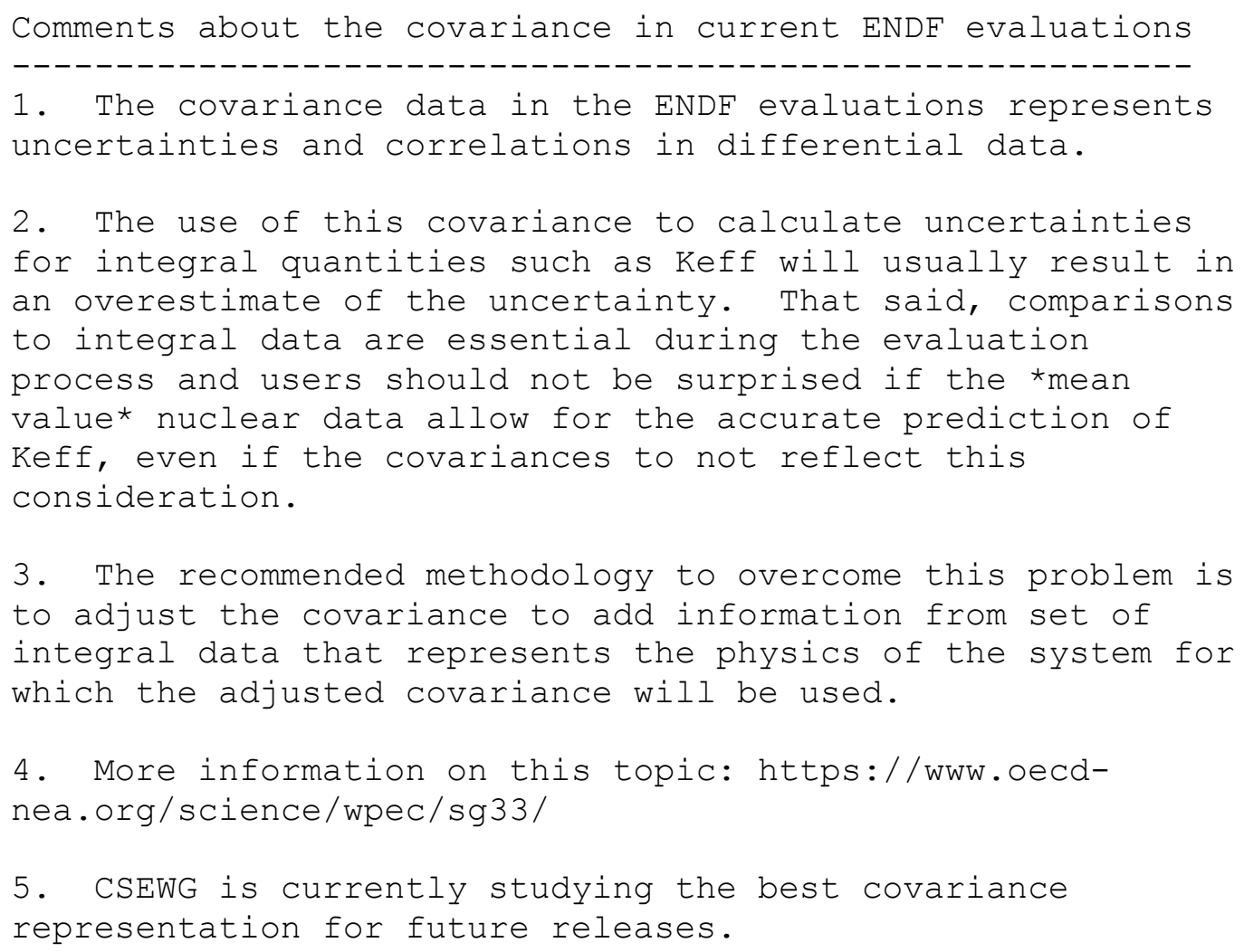

As has been discussed above, this disclaimer leaves no ambiguity of the interpretation of the covariance data provided. However, from this statement, it is also clear that what is provided in the ENDF/B-VIII.0 library is not self-consistent. The mean values of the nuclear data have been adjusted while the covariance data does not reflect this increase in knowledge. It is the goal of the work, reported in Section 5 , to provide a first estimate of how the covariance data should be augmented to reflect the adjustment of the mean values. 


\section{ENDF/B-VIII.0 COVARIANCE TESTING WITH INTEGRAL BENCHMARK EXPERIMENTS}

The recent release of ENDF/B-VIII.0 [2] contains new covariance data for many isotopes. The covariance data have been processed at ORNL into a beta covariance library for testing and evaluation. A range of testing is conducted to investigate the properties of these covariance data and ensure that the data are reasonable. These tests include examination of the uncertainty in critical experiment benchmark model and application model $\mathrm{k}_{\text {eff }}$ values due to nuclear data uncertainties, as well as similarity assessments of irradiated pressurized water reactor (PWR) fuel with a suite of critical experiments. The contents of the new covariance library, the testing performed, and the behavior of the new covariance data are described here. Testing applicable to advanced reactor models is described in Section 4.

The neutron cross-section covariances can be combined with a sensitivity data file generated using the TSUNAMI suite of codes within SCALE to determine the uncertainty in system $k_{\text {eff }}$ caused by nuclear data uncertainties [3]. The Verified, Archived Library of Inputs and Data (VALID) maintained at ORNL contains sensitivity data for over 400 critical experiment benchmark models [4]. The nuclear data uncertainty in $k_{\text {eff }}$ is generated for each experiment, and the resulting uncertainties are tabulated and compared to the differences in measured and calculated results.

One of the primary applications of sensitivity/uncertainty methods within SCALE is the assessment of similarity between benchmark experiments and safety applications, as described by the $\mathrm{c}_{\mathrm{k}}$ value for each experiment with each application [3]. Several studies have analyzed typical $c_{k}$ values for a range of critical experiments compared with hypothetical irradiated fuel applications $[5,6]$. The $c_{\mathrm{k}}$ value is sensitive to the cross-section covariance data because the contribution of each nuclide is influenced by its uncertainty; large uncertainties indicate more likely bias sources and are thus given more weight. Changes in $\mathrm{c}_{\mathrm{k}}$ values resulting from different covariance data can be used to examine and assess underlying data changes. These comparisons are performed for PWR fuel in a storage and transportation system.

\subsection{COVARIANCE LIBRARIES}

Testing of the ENDF/B-VIII.0 covariance library is largely performed by comparison with the covariance library deployed with SCALE 6.2 [7]. A brief description of both the SCALE 6.2 and ENDF/B-VIII.0 covariance libraries is provided below. A selection of importance isotopes and reactions are also compared later in this section.

\subsubsection{SCALE 6.2 COVARIANCE LIBRARY}

The SCALE 6.2 covariance library has incorporated ENDF/B-VII.1 covariance data for over 180 nuclides. These evaluations are used, with the exception of the data for three isotopes for which testing revealed problems in the data: ${ }^{1} \mathrm{H},{ }^{235} \mathrm{U}$, and ${ }^{239} \mathrm{Pu}[6]$. A few high-fidelity evaluations from ENDF/B-VI remain in the library. New covariance data were generated for fission spectra based on the data contained in ENDF/B File 35. As with previous SCALE covariance libraries, the remaining covariance data provided are the low-fidelity evaluations documented in Ref. [8]. The SCALE 6.2 covariance data are tabulated in 56 groups instead of the 44 used in the SCALE 6.1 library. TSUNAMI-IP [7] allows the use of existing 238-group sensitivity data files (SDFs) with the 56-group uncertainty data, so existing SDFs can still be used with the SCALE 6.2 library.

\subsubsection{ENDF/B-VIII.0 EXPERIMENTAL COVARIANCE LIBRARY}

The SCALE experimental ENDF/B-VIII.0 covariance library has been created by processing all available covariance evaluations. Most other data are taken from the SCALE 6.2 covariance library. Some new fission spectrum data are also included from JENDL evaluations. 


\subsubsection{MAJOR ISOTOPES AND REACTIONS}

Most of the systems of interest containing fissile material are fueled by either ${ }^{235} \mathrm{U}$ or ${ }^{239} \mathrm{Pu}$ and the primary moderator of interest is ${ }^{1} \mathrm{H}$. The uncertainties on the fission cross sections and average number of neutrons produced per fission (nu-bar) for both ${ }^{235} \mathrm{U}$ and ${ }^{239} \mathrm{Pu}$ are therefore of great interest. The covariance data for scattering in ${ }^{1} \mathrm{H}$ is similarly of significant interest. Plots of the uncertainty data for these isotope/reaction pairs is provided in this section, along with a brief discussion of each figure.

The uncertainty in the ${ }^{1} \mathrm{H}$ elastic scattering reaction is provided in Figure 5 for both the SCALE 6.2 and ENDF/B-VIII.0 covariance libraries. Although cross sections between the two evaluations are virtually entirely inconsistent, the uncertainty up to the $\mathrm{keV}$ range has increased from approximately $0.14 \%$ to around $0.84 \%$. This dramatic change will, all else being equal, increase the reported uncertainty in thermal, water-moderated systems such as LWRs. Furthermore, in the SCALE 6.2 data, the uncertainty increases above $1 \mathrm{keV}$ to a maximum of around $1 \%$ at approximately $5 \mathrm{MeV}$. The ENDF/B-VIII.0 covariance, on the other hand, drops above $1 \mathrm{keV}$ to a minimum of approximately $0.4 \%$ at about $1 \mathrm{MeV}$. Large changes in covariance evaluations are fairly common between ENDF releases and degrade the ability to rely on uncertainty quantification to gauge the predictive nature of simulations.

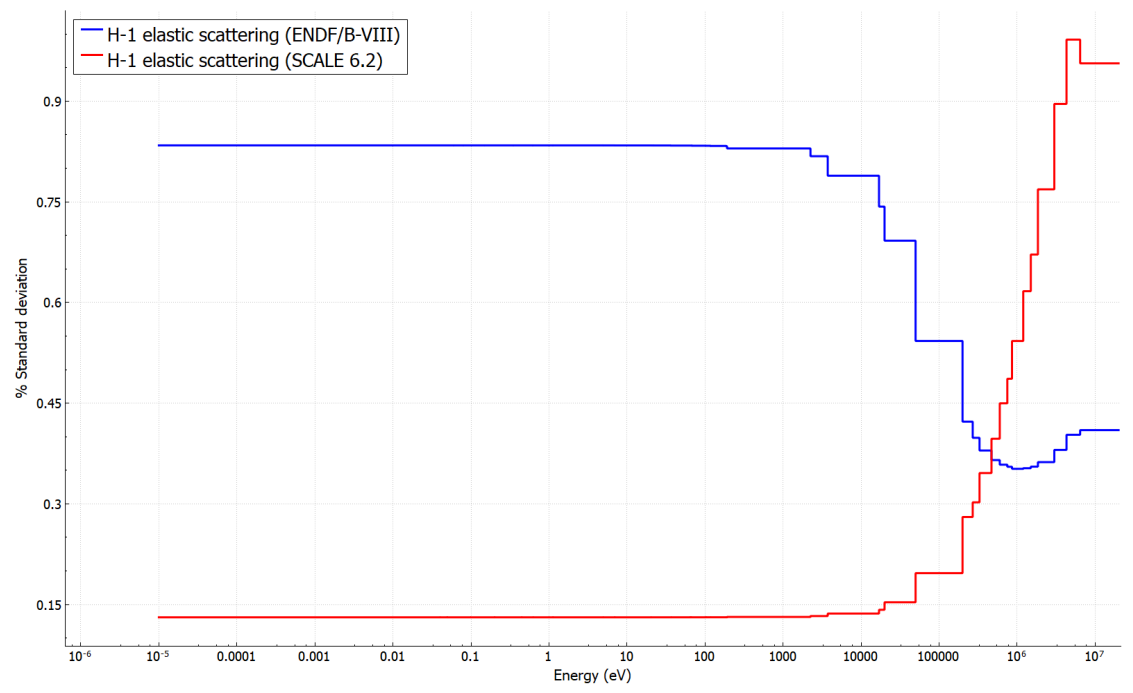

Figure 5. Uncertainty in ${ }^{1} \mathrm{H}$ elastic scattering.

The uncertainty in the fission reaction for ${ }^{235} \mathrm{U}$ is shown in Figure 6 and for nu-bar in Figure 7. The uncertainty in the fission reaction increases across almost the entire energy range. The uncertainty nearly doubles between 0.1 and $1 \mathrm{eV}$ and nearly triples above about $50 \mathrm{keV}$. The only noticeable decrease between the SCALE 6.2 data and the ENDF/B-VIII.0 data is between approximately 200 and $1200 \mathrm{eV}$. The uncertainty in nu-bar also increases across the entire energy range. The ENDF/B-VIII.0 uncertainty is higher at all energies. The increase is small, from about $0.37 \%$ in the SCALE 6.2 data to approximately $0.47 \%$ in the ENDF/B-VIII.0 data. Above about $100 \mathrm{keV}$, the uncertainty in both libraries has a similar shape, but the ENDF/B-VIII.0 uncertainty is nearly twice the SCALE 6.2 uncertainty of around $0.25 \%$. There is also a significant increase in the uncertainty in the intermediate energy range in the ENDF/BVIII.0 evaluation, and features in this range that are entirely absent in the SCALE 6.2 data. The changes in the nu-bar uncertainty appear to be more dramatic than those in the fission uncertainty. 


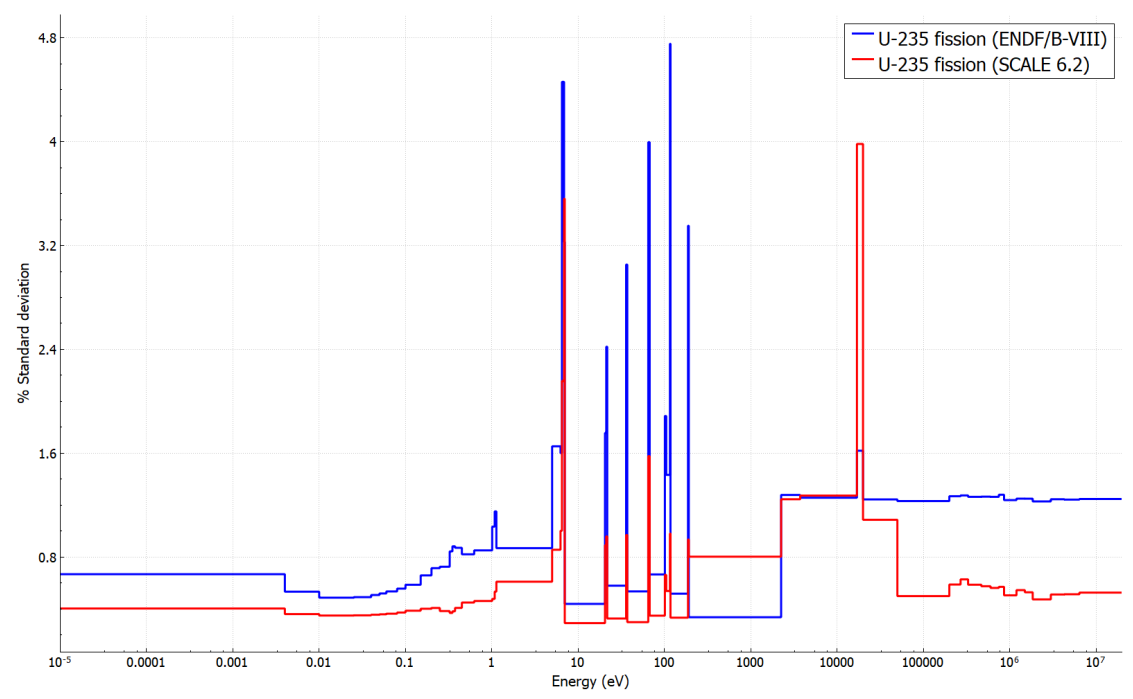

Figure 6. Uncertainty in $235 \mathrm{U}$ fission

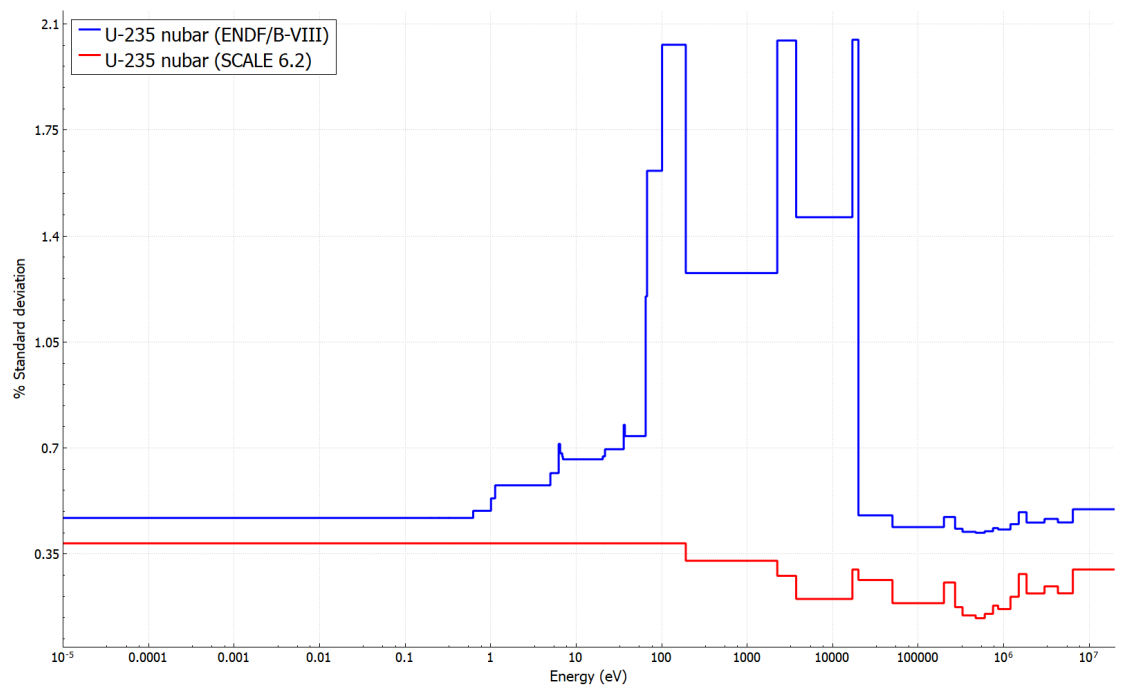

Figure 7. Uncertainty in ${ }^{235} \mathrm{U}$ nu-bar

The uncertainty in ${ }^{239} \mathrm{Pu}$ fission and nu-bar are provided in Figures 8 and 9 . The uncertainties in the fission cross section are qualitatively quite different between the SCALE 6.2 data and the ENDF/B-VIII.0 data. The magnitude of the differences is smaller than it has been for some of the other reactions. The uncertainty over the range from about 0.025 to $1 \mathrm{eV}$ is higher in the ENDF/B-VIII.0 evaluation than in the SCALE 6.2 data. This range is particularly important for thermal systems; the energy of the average lethargy causing fission (EALF) for the thermal Pu solution systems in VALID varies from about 0.05 to $0.34 \mathrm{eV}$. The peak difference between the two libraries in the thermal range is almost a factor of 3 at an energy of about $0.2 \mathrm{eV}$. The ratio in the fast range has ENDF/B-VIII.0 with a little more than twice the uncertainty than the SCALE 6.2 data. This energy range is important to fast metal systems. As with ${ }^{235} \mathrm{U}$, the nu-bar uncertainty for ${ }^{239} \mathrm{Pu}$ is higher for ENDF/B-VIII.0 at all energies. The uncertainty from 0.01 to about $50 \mathrm{eV}$ is approximately $0.185 \%$ in the SCALE 6.2 data and just over $0.32 \%$ in the ENDF/B-VIII.0 evaluation. Similar differences exist around $1 \mathrm{MeV}$, and large differences are present for higher energies. 


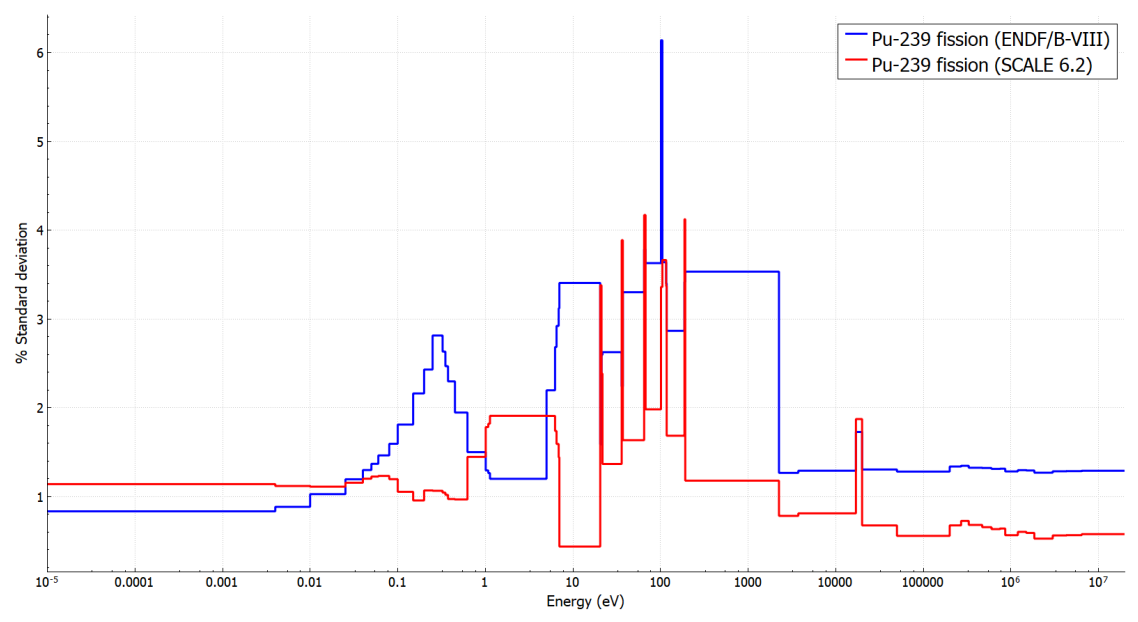

Figure 8. Uncertainty in ${ }^{239} \mathrm{Pu}$ fission

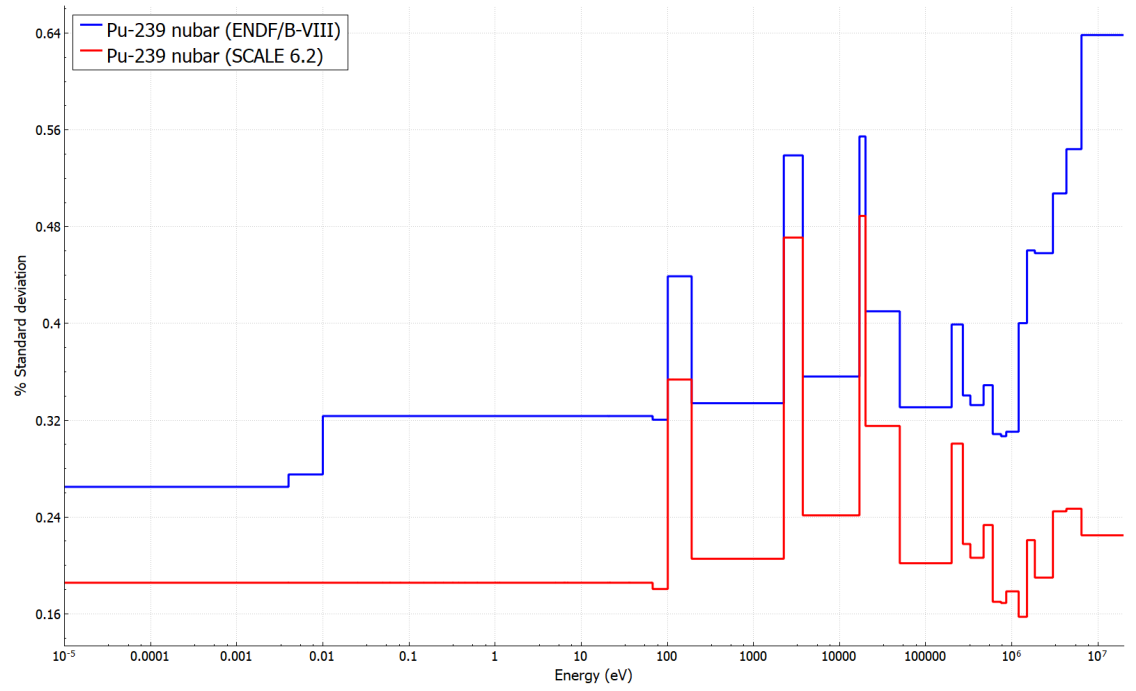

Figure 9. Uncertainty in ${ }^{239} \mathrm{Pu}$ nu-bar

\subsection{NUCLEAR DATA-INDUCED UNCERTAINTY IN VALID MODEL $K_{\text {EFF }}$ VALUES}

The first test method considers the predicted uncertainty in $k_{\text {eff }}$ for critical benchmark experiment models resulting from cross-section uncertainty. In this work, this uncertainty is determined through the use of TSUNAMI-IP [7] and available SDFs from VALID [4]. The existing sensitivities are propagated with the both sets of covariance data to the uncertainty in $k_{\text {eff; }}$ the details of the method are described in Ref. [3]. A comparison of the distribution of the calculated-to-experiment $(\mathrm{C} / \mathrm{E})$ ratios to this uncertainty band for these experiments provides an indication of the accuracy of the cross-section-induced $k_{\text {eff }}$ uncertainty. For comparison, the distribution of $\mathrm{C} / \mathrm{E}$ values is also compared with the uncertainty in the critical experiment evaluation.

A series of comparisons of the new and old cross-section uncertainty bands is shown in Figures 10, 11, 13 and 14. The $k_{\text {eff }} \mathrm{C} / \mathrm{E}$ and its uncertainty, dominated by the experimental uncertainty, are shown for each case. The SCALE 6.2 covariance band is shown as a black line with long dashes and the ENDF/B-VIII.0 uncertainty band is provided in a red line with shorter dashes. 


\subsubsection{HEU-MET-FAST SYSTEMS}

The results for fast, high-enriched uranium metal (HMF) systems are shown in Figure 10. It is immediately obvious that there are significant differences between the $k_{\text {eff }}$ uncertainties for these systems. The ENDF/B-VIII.0 results show a larger variation, with uncertainties ranging from just under $1 \%$ to over $5 \%$. For comparison, the SCALE 6.2 covariance data yields $k_{\text {eff }}$ uncertainties ranging from just over $1 \%$ to just under $2.5 \%$.

The first case on the plot corresponds to the HMF-015 evaluation and shows good overall agreement in data induced uncertainty in $k_{\text {eff. }}$. A detailed review of the contributions to this uncertainty, however, show that the top contributors to uncertainty are different between the two libraries. The top contributors in the SCALE 6.2 library are ${ }^{235} \mathrm{U}$ absorption and scattering. The top contributors based on the ENDF/B-VIII.0 data are ${ }^{235} \mathrm{U}$ fission and nu-bar. The uncertainty contribution from ${ }^{235} \mathrm{U}$ capture drops almost two thirds, while the contributions from fission and nu-bar increase by factors of 3 and 4.7 respectively.

The 7th and 8th point on the plot correspond to detailed and simplified models of the HMF-019 evaluation. Both models represent the same experiment with different levels of fidelity, but the sensitivity data are similar in both cases, so the impacts of the covariance data are also very similar. These cases show the largest discrepancy between the SCALE 6.2 and ENDF/B-VIII.0 data. The data induced uncertainty in $k_{\text {eff }}$ using the SCALE 6.2 covariance data is $1.18 \%$ but is $5.19 \%$ using ENDF/B-VIII.0 data. Nearly all of this huge uncertainty predicted with the ENDF/B-VIII.0 data is a result of using a default estimate of the covariance data for graphite in this graphite-reflected system. The default covariance data is a $40 \%$ uncertainty in the fast energy range, compared to an uncertainty of just under $0.8 \%$ at $1 \mathrm{MeV}$ in the SCALE 6.2 data. It is possible that the default data is used because of differences in the treatment of graphite between ENDF/B-VII.1 and ENDF/B-VIII.0. Graphite is represented in isotopic, as opposed to elemental, form for the first time in ENDF/B-VIII.0. This points to challenges encountered with both the processing codes (e.g., AMPX) and the analysis codes (e.g., TSUNAMI-IP) in properly using covariance data and sensitivity data from different evaluations. ENDF/B-VIII.0 and the appropriate material identifiers will be included in the upcoming release of SCALE 6.3. Similar assessments can be performed to explain the cause of each of the differences between the SCALE 6.2 and ENDF/B-VIII.0 results, but such analyses have not been performed at this writing.

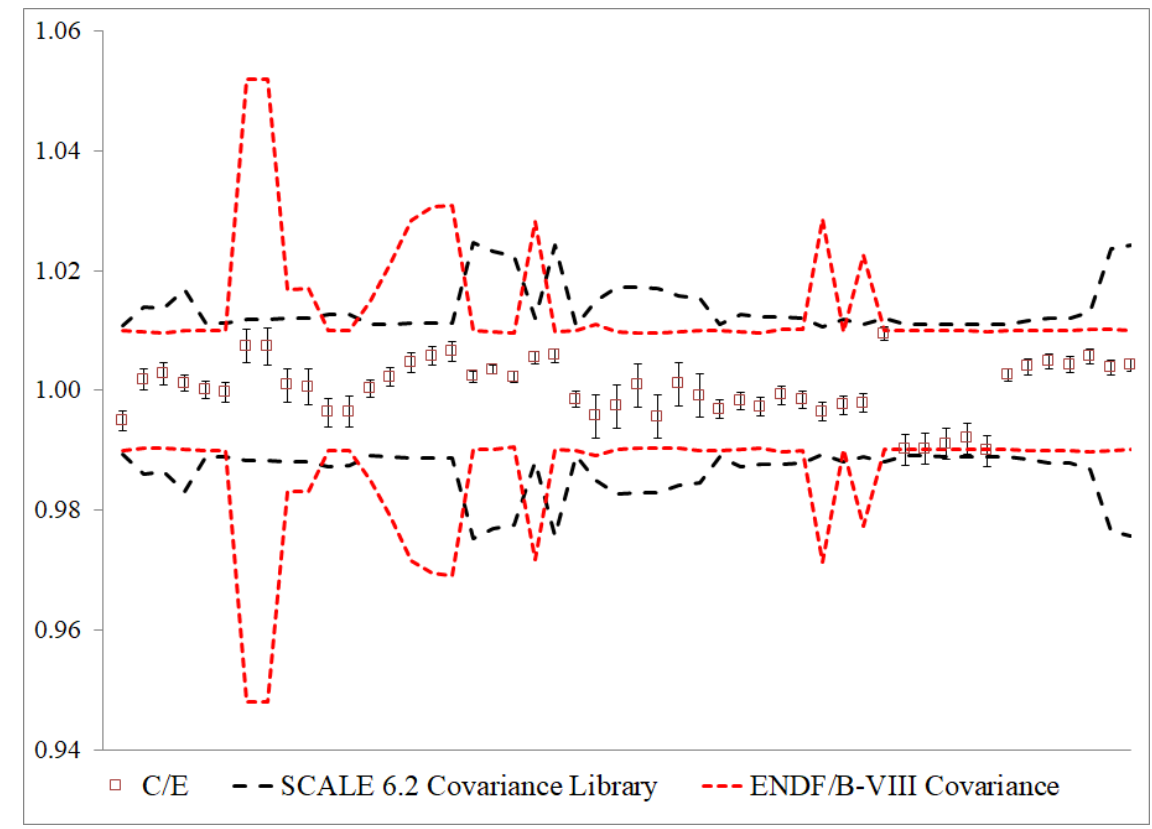

Figure 10. Results for HEU-MET-FAST systems. The experiments are sorted by their ICBSEP-assigned number on the horizontal axis. $\mathrm{C} / \mathrm{E}$ values are shown on the vertical axis. 


\subsubsection{LEU-COMP-THERM SYSTEMS}

The results for thermal, low-enriched uranium compound (LCT) systems are shown in Figure 11. The results are much more similar for these systems than for the HMF systems discussed in the previous section. As with the case of HMF-015-001 discussed in the previous section, the similar overall data induced uncertainty is a fortuitous balancing of several different changes and not the result of similar covariance evaluations.

The first case in Figure 11 is LCT-001-001, and the data induced uncertainty in $k_{\text {eff }}$ is $0.69 \%$ for the SCALE 6.2 covariance library and $0.66 \%$ for the ENDF/B-VIII.0 data. In both cases, ${ }^{235} \mathrm{U}$ nu-bar is the top uncertainty contributor, but it contributes $0.36 \%$ with the SCALE 6.2 data and $0.44 \%$ in ENDF/BVIII.0. It is important to note in these discussions that the individual uncertainty contributions are combined using the square root of the sum of the squares, so the top contributors are by far the most important. The second highest contributor with the SCALE 6.2 data is the uncertainty in the energy distribution of fission neutrons $(\chi)$, but its contribution of $0.31 \%$ is slashed to $0.05 \%$ with the ENDF/BVIII.0 data. This is a result of an uncertainty that is approximately 2 to 3 times higher in the SCALE 6.2 data over the energy range in which most fission neutrons are born. The third highest contributor to uncertainty with the SCALE 6.2 data is inelastic scatter on ${ }^{238} \mathrm{U}$, with an uncertainty contribution of $0.26 \%$. The same uncertainty contribution with the ENDF/B-VIII.0 is $0.04 \%$. This is a result of massive changes in the covariance data for this reaction, as shown in Figure 12. The highest cross section for ${ }^{238} \mathrm{U}$ inelastic scattering is above $1 \mathrm{MeV}$, where the uncertainty is 2 to 5 times higher in the SCALE 6.2 data. The reductions in the uncertainty of ${ }^{235} \mathrm{U}$ nu-bar, fission, and $\chi$ offset large increases in the ${ }^{1} \mathrm{H}$ uncertainties in scatter and absorption in the ENDF/B-VIII.0 data. The energy dependent uncertainties in elastic scatter are shown in Figure 5 in Section 3.1.3. The uncertainty in $k_{\text {eff }}$ in LCT-001-001 increases modestly from $0.11 \%$ with SCALE 6.2 data to $0.19 \%$ with ENDF/B-VIII.0 data; the elastic scattering uncertainty is the 11th highest contribution with the SCALE 6.2 data but the third highest with the ENDF/B-VIII.0 covariances. The uncertainty contribution from ${ }^{1} \mathrm{H}$ capture increases from $0.21 \%$ to $0.4 \%$, and the reaction moves from the 5 th to the 2 nd largest contributor to uncertainty.

The case of LCT-078-015 is also examined because this is the largest difference between the data induced uncertainty estimates from the two covariance data sets. The primary reactions contributing to uncertainty are mostly the same as those discussed in the previous case regarding LCT-001-001. In this case, however, the uncertainty contributions from ${ }^{235} \mathrm{U} \chi$ and ${ }^{238} \mathrm{U}$ inelastic scatter are significantly larger than for LCT-001-001. The reduction in the uncertainty for these covariance data in the ENDF/B-VIII.0 overwhelm the increases in ${ }^{1} \mathrm{H}$. The total data induced uncertainty in $k_{\text {eff }}$ is thus lowered from $0.84 \%$ with the SCALE 6.2 covariance data to $0.59 \%$ using the ENDF/B-VIII.0 data. This case illustrates that the differences between the covariance data can have different impacts even within fairly similar systems. 


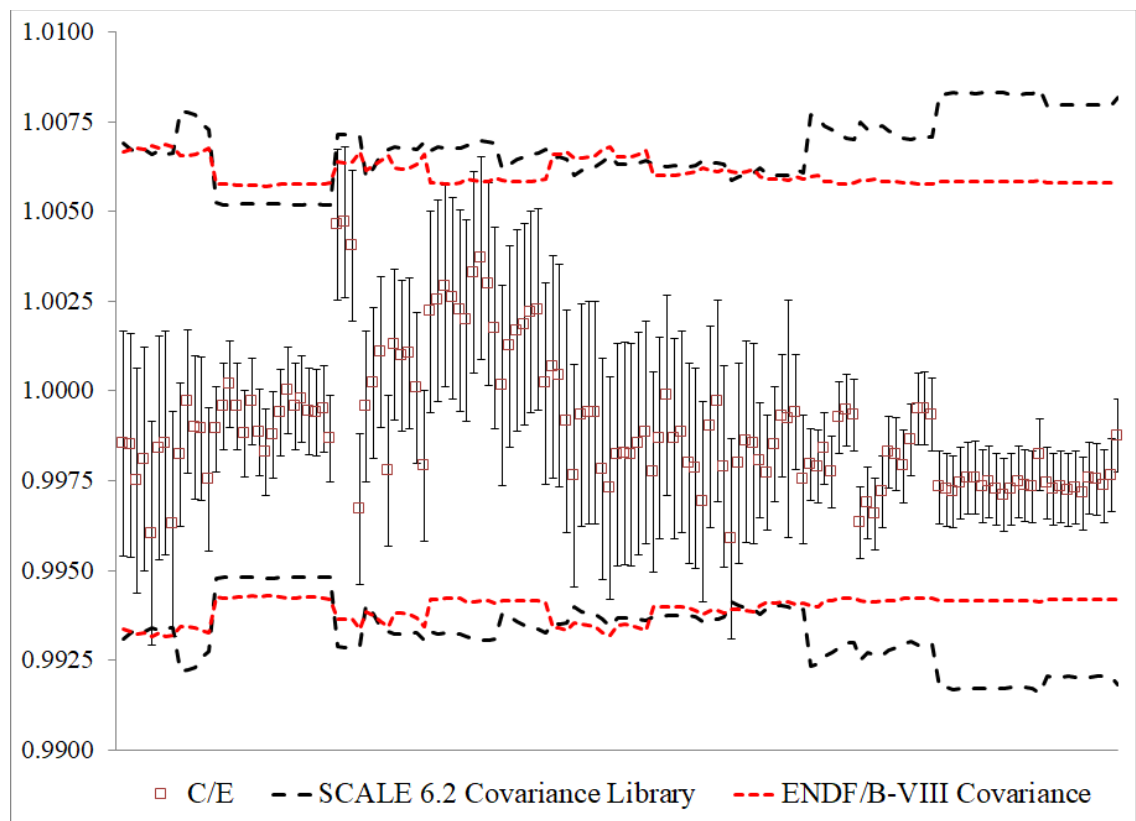

Figure 11. Results for LEU-COMP-THERM systems. The experiments are sorted by their ICBSEP-assigned number on the horizontal axis. $\mathrm{C} / \mathrm{E}$ values are shown on the vertical axis.

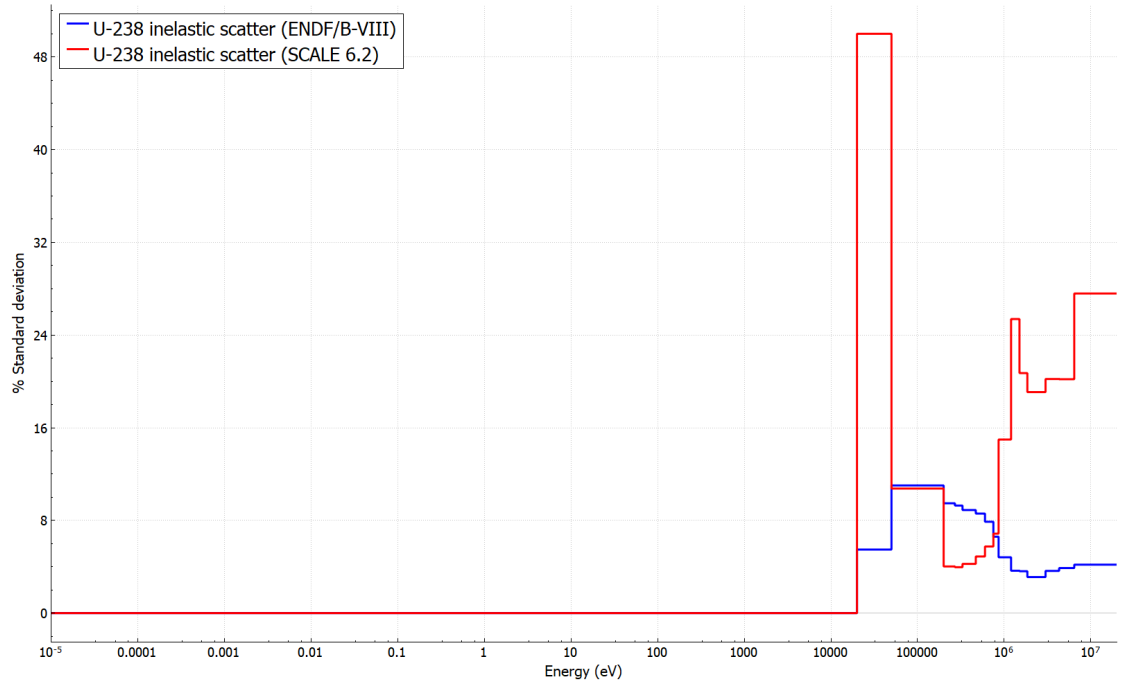

Figure 12. Uncertainty in ${ }^{238} \mathrm{U}$ inelastic scattering

\subsubsection{MIX-COMP-THERM SYSTEMS}

The results for thermal, mixed uranium and plutonium oxide compound (MCT) systems are shown in Figure 13. The results show a clear increase in the data induced uncertainty using the ENDF/B-VIII.0 covariance data. The increase in uncertainty ranges from $0.30 \%$ to $0.54 \%$, with 41 of the 49 cases experiencing an uncertainty increase of between $0.30 \%$ and $0.35 \%$. This category of systems is important because it is representative of spent LWR fuel. 
MCT-008-002 is selected for detailed investigation; the data induced uncertainty in $k_{\text {eff }}$ for this experiment is $0.62 \%$ using the SCALE 6.2 covariance data and $0.93 \%$ with the ENDF/B-VIII.0 data. This difference, as discussed previously, is typical for the MCT cases in VALID. The top two contributors to uncertainty using the SCALE 6.2 data are ${ }^{239} \mathrm{Pu}$ fission $(0.297 \%)$ and ${ }^{238} \mathrm{U}$ inelastic scattering $(0.295 \%)$. The ${ }^{239} \mathrm{Pu}$ fission reaction is the second highest contribution to uncertainty using the ENDF/B-VIII.0 data, but the magnitude of the uncertainty jumps to $0.50 \%$. The largest uncertainty contribution for the ENDF/B-VIII.0 data is ${ }^{239} \mathrm{Pu}$ capture, which induces a $k_{\text {eff }}$ uncertainty of $0.79 \%$. In other words, the uncertainty in $k_{\text {eff }}$ caused by uncertainty in the ENDF/B-VIII.0 evaluation of ${ }^{239} \mathrm{Pu}$ capture is larger than all the data induced uncertainty from the SCALE 6.2 library. The change in ${ }^{239} \mathrm{Pu}$ nu-bar uncertainty is shown in Figure 8; the large increase between 0.1 and $1 \mathrm{eV}$ is largely responsible for the uncertainty increase in these thermal systems. The ${ }^{1} \mathrm{H}$ elastic scattering and capture effects discussed in the previous section are also present with the MCT systems and contribute to the increase in data induced uncertainty. The ${ }^{239} \mathrm{Pu}$ nu-bar contribution also increases to $0.23 \%$ with the ENDF/B-VIII.0 data from $0.13 \%$ with the SCALE 6.2 covariance data; the energy dependent uncertainty for ${ }^{239} \mathrm{Pu}$ nu-bar is shown in Figure 9.

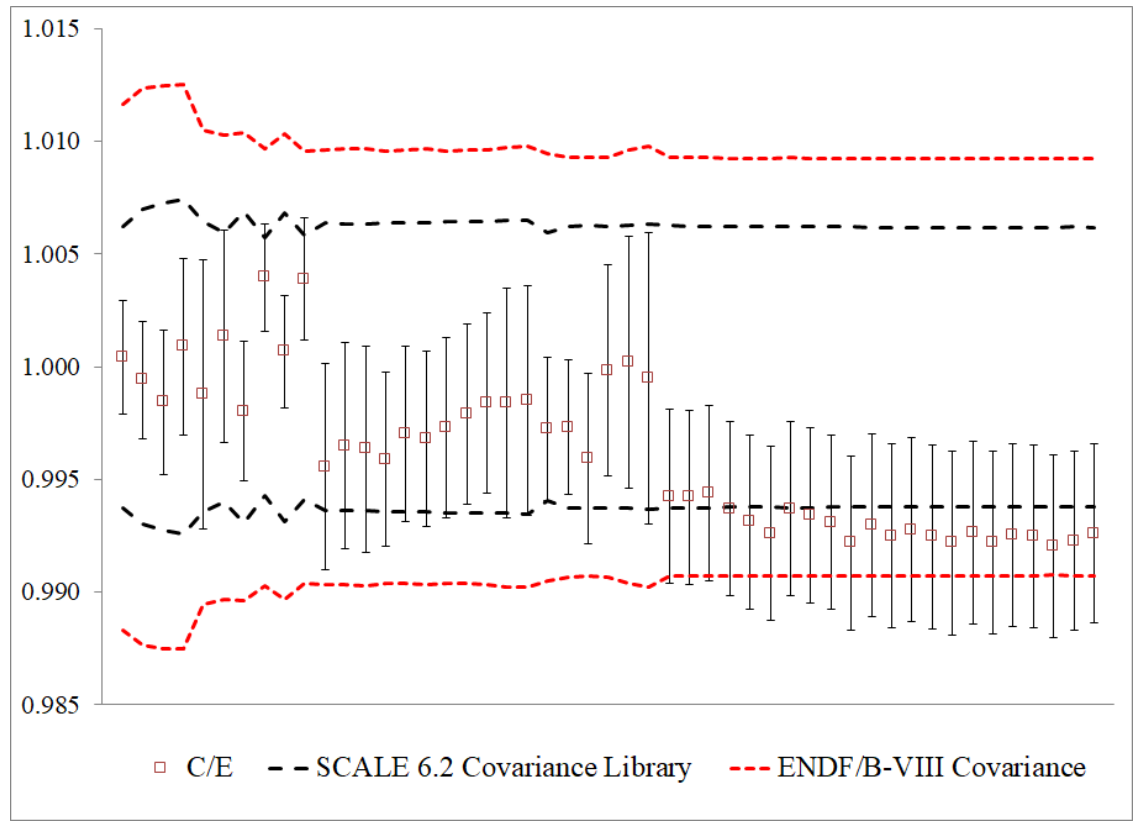

Figure 13. Results for the MIX-COMP-THERM systems. The experiments are sorted by their ICBSEPassigned number on the horizontal axis. $\mathrm{C} / \mathrm{E}$ values are shown on the vertical axis.

\subsubsection{PU-MET-FAST SYSTEMS}

The results for fast, plutonium metal (PMF) systems are shown in Figure 14. The results appear to show a similar level of data induced uncertainty for 10 or the 11 cases. The massive difference in the one case distorts the scale of the plot; the data induced uncertainty increases significantly, on the order of doubling, for most cases. The one extreme increase is for PMF-023, which is a graphite reflected system; the problem with using isotopic graphite covariance data was mentioned with respect to HMF-019 in Section 3.2.1 and is the cause of the outlier here as well.

A detailed analysis of the data induced uncertainty in $k_{\text {eff }}$ for PMF-001 is presented here. The top three uncertainty contributions for PMF-001 using the SCALE 6.2 covariance data are inelastic scattering, elastic scattering, and the correlation between these reactions. These same contributors, with similar magnitudes, occur with the ENDF/B-VIII.0 data. The uncertainty in ${ }^{239} \mathrm{Pu}$ fission jumps from the fourth most important reaction, with an uncertainty contribution of $0.33 \%$ using the SCALE 6.2 data, to the most important uncertainty. With the ENDF/B-VIII.0 covariance data the uncertainty in $k_{\text {eff }}$ contributed by 
${ }^{239} \mathrm{Pu}$ fission is $0.91 \%$. The uncertainty in ${ }^{239} \mathrm{Pu}$ nu-bar also increases from $0.08 \%$ with SCALE 6.2 data to $0.32 \%$ using the ENDF/B-VIII.0 covariance data. As with the MCT systems discussed in Section 3.2.3, the increased uncertainty in the Pu reactions leads to an overall increase in the data induced uncertainty in $k_{\text {eff. }}$

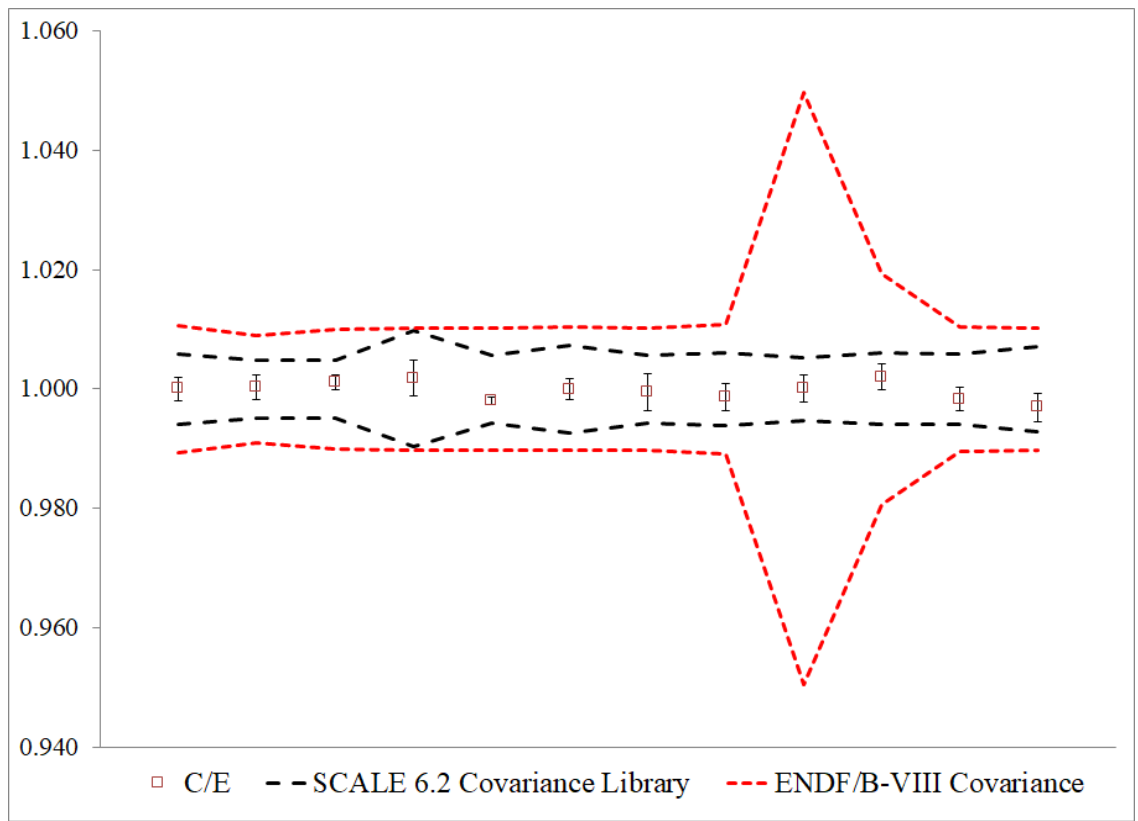

Figure 14. Results for PU-MET-FAST systems. The experiments are sorted by their ICBSEP-assigned number on the horizontal axis. $C / E$ values are shown on the vertical axis.

\subsubsection{SUMMARY OF ALL CATEGORIES}

Ten difference categories of experiments have sensitivity data in the VALID library and can be used to assess covariance data by comparing the distribution of $\mathrm{C} / \mathrm{E}$ values to the data induced $k_{\text {eff }}$ uncertainties predicted with various nuclear data covariance libraries. Detailed assessments of four of these categories have been presented in the previous subsections of Section 3.2. A summary of the results for all 10 categories is provided in Table 1. The standard deviation of the $\mathrm{C} / \mathrm{E}$ values is presented along with the average data induced uncertainties using both the SCALE 6.2 and the ENDF/B-VIII.0 covariance libraries. The fraction of $\mathrm{C} / \mathrm{E}$ values within one standard deviation of unity is also included based on the experimental uncertainty from the evaluation and based on the $k_{\text {eff }}$ uncertainty induced by the ENDF/BVIII.0 covariance data.

It is expected that $68.3 \%$ of the population of a normally distributed data set would be within one standard deviation of the average. With respect to the experimental uncertainties, three of the 10 categories of experiments have a larger portion of the population within one standard deviation than expected, 6 categories have fewer than the expected number of results in this range, and one category is essentially exactly at the fraction. All ten categories have a larger portion of the population than expected within one standard deviation based on the data induced uncertainty, and 7 of the categories have all points within one standard deviation of the mean. Two additional categories have only 1 case outside the single standard deviation from the data induced $k_{\text {eff }}$ uncertainty. The standard deviation of the C/E values is approximately the same as the average experimental uncertainty for most categories. The ratio of the standard deviation of the $\mathrm{C} / \mathrm{E}$ values to the average experimental uncertainty ranges from 0.4 to 1.6 , with the ratio for 6 of the 10 categories between 0.7 and 1.3. The average data induced uncertainty exceeds the standard deviation of the $\mathrm{C} / \mathrm{E}$ values for all 10 categories of experiments by a factor ranging from 1.1 to 11.4. For most of the categories, the data induced uncertainty exceeds the actual $\mathrm{C} / \mathrm{E}$ variation by a factor 
of 3-4. The indication of these results is that the nuclear covariance data overestimate the data induced uncertainty in $k_{\text {eff. }}$.

Table 1. Uncertainty Summary for Ten Categories of Experiments in VALID (Uncertainties are reported in pcm).

\begin{tabular}{ccccccccc}
\hline Category & $\begin{array}{c}\text { Avg. Exp. } \\
\text { Unc. }\end{array}$ & $\begin{array}{c}\text { St Dev of } \\
\text { C/E Values }\end{array}$ & $\begin{array}{c}\text { SCALE 6.2 } \\
\text { XS Unc }\end{array}$ & $\begin{array}{c}\text { ENDF/B8 } \\
\text { XS Unc }\end{array}$ & $\begin{array}{c}\text { ENDF/B8 } \\
\text { Augmented } \\
\text { XS Unc }\end{array}$ & $\begin{array}{c}\text { \% Cases wrt } \\
\text { Exp Unc }\end{array}$ & $\begin{array}{c}\text { \% Cases wrt } \\
\text { ENDF/B8 } \\
\text { XS Unc }\end{array}$ & $\begin{array}{c}\text { \%oses wrt } \\
\text { Aug. E8 } \\
\text { XS Unc }\end{array}$ \\
\hline HMF & 193 & 476 & 1,380 & 1,442 & 1121 & 50.0 & 98 & 90 \\
HST & 494 & 593 & 1,051 & 655 & 611 & 42.3 & 75 & 75 \\
IMF & 269 & 359 & 1,522 & 1,593 & 1496 & 30.8 & 100 & 100 \\
LCT & 195 & 167 & 677 & 606 & 557 & 58.6 & 100 & 100 \\
LST & 318 & 264 & 716 & 824 & 775 & 68.4 & 100 & 100 \\
MCF & 220 & 478 & 1,215 & 1,049 & 980 & 50 & 100 & 100 \\
MCT & 400 & 337 & 633 & 973 & 958 & 46.9 & 100 & 100 \\
MST & 452 & 293 & 854 & 1,323 & 1302 & 80 & 100 & 100 \\
PMF & 207 & 126 & 621 & 1,425 & 1248 & 83.3 & 100 \\
PST & 497 & 423 & 851 & 1,344 & 1326 & 75.3 & 98.8 \\
\hline
\end{tabular}

\subsection{SIMILARITY ASSESSMENT}

The next component of the covariance data testing is determining the $c_{k}$ values for a number of experiments compared to spent PWR fuel in a storage and transportation system model. The integral index $c_{k}$ is a correlation coefficient for each experiment with each application based on the sensitivities propagated with the covariance data. It is calculated by determining the amount of nuclear data induced uncertainty that is shared between two systems. A full description of the calculation of $c_{k}$ is provided in Ref. [3]. The covariance data effectively act as a weighting function for the sensitivities; the nuclide/reaction pairs with larger uncertainties contribute more strongly to $c_{k}$ because they are expected to be larger sources of bias since they are more uncertain. Typically, a $c_{k}$ value of 0.8 or higher indicates sufficient similarity between the experiment and the application to allow the application to be used for validation [3]. This threshold value of 0.8 is shown in Figure 15 for reference, although the primary focus of this section is the change in $c_{k}$ caused by the use of the SCALE 6.2 or the ENDF/B-VIII.0 covariance library. The $c_{k}$ values are also compared to those resulting from the use of the SCALE 6.1 covariance library. This library is used only in this section because it was the basis for the analyses [5] which support the US Nuclear Regulatory Commission's Division of Spent Fuel Storage and Transportation Interim Staff Guidance 8, Revision 3 [9]; therefore, a comparison to the ENDF/B-VIII.0 results may be relevant in regulatory proceedings.

A collection of SDFs for 1,643 experiments was created for work documented in Ref. [10]. The SDFs are drawn from VALID, cases used in Ref. [5], and SDFs generated by the Nuclear Energy Agency [11]. The set of experiments includes over 1,000 LCT experiments and over $250 \mathrm{MCT}$ experiments. The set also includes the Haut Taux de Combustion (HTC) experiments [12], a series of MCT experiments with the actinide composition designed to match spent fuel, and a collection of low-enriched uranium and mixed uranium/plutonium (MIX) solution cases. The last component of the experiment set is a group of cases categorized as miscellaneous configurations with low-enriched uranium fissile material. A complete list of the experiments is provided in Ref. [11], Appendix C. The results are shown in Figure 15 for the comparison of the ENDF/B-VIII.0 data with the SCALE 6.2 covariance library and in Figure 16 for 
comparison with the SCALE 6.1 library. In both figures, the results based on ENDF/B-VIII.0 are shown in black.

The results show that the HTC experiments are still the most applicable experiments for validation of spent PWR systems. This is the expected result because the HTC actinide material was specifically fabricated to represent PWR fuel with a typical discharge burnup. The applicability of some MCT experiments has increased while that of others has decreased. The experiments with lower $c_{k}$ values contain depleted uranium and are moderated with polystyrene, which the experiments with improved applicability has natural uranium and are water moderated. The variation of applicability of LCT systems has, in general, been reduced compared to the SCALE 6.2 covariance data. Some LCT experiments have significantly lower applicability; as with the MCT experiments with low $c_{k}$ values, these systems are moderated with a material other than water, typically wax or plastic. Some of the reduction in the $c_{k}$ values appears to be related to difficulty in mapping the ${ }^{1} \mathrm{H}$ covariance data to the hydrogen present in both the MCT and LCT systems, but this effect appears to be less than approximately 0.05 on the $c_{k}$ values. The applicability of the mixed uranium/plutonium solution systems is significantly higher with the ENDF/B-VIII.0 data than with the SCALE 6.2 data.

The comparison of $c_{k}$ values between the SCALE 6.1 covariance data used in [5] and the ENDF/B-VIII.0 covariance data is shown in Figure 16. The results of the applicability determinations using these two libraries is generally quite good. The water-moderated LCT systems show higher applicability because of increased uncertainties in ${ }^{235} \mathrm{U}$ in ENDF/B-VIII.0 compared to the SCALE 6.1 data. Most of the watermoderated MCT systems have very similar $\mathrm{c}_{\mathrm{k}}$ values; the polystyrene-moderated MCT systems with depleted uranium show significantly lower applicability. This is most likely a result of the lower uncertainty in ${ }^{238} \mathrm{U}$ inelastic scattering in the ENDF/B-VIII.0 data. The HTC $\mathrm{c}_{\mathrm{k}}$ values are largely equivalent, and these systems continue to show the highest applicability to the PWR spent fuel system. The effect of the ENDF/B-VIII.0 data on experiment applicability in PWR burnup credit is largely a return to the SCALE 6.1 assessments. The details of the applicability determinations differ significantly from those made with the SCALE 6.1 covariance data, but the differences largely cancel and suggest the same types of experiments are useful for validation.

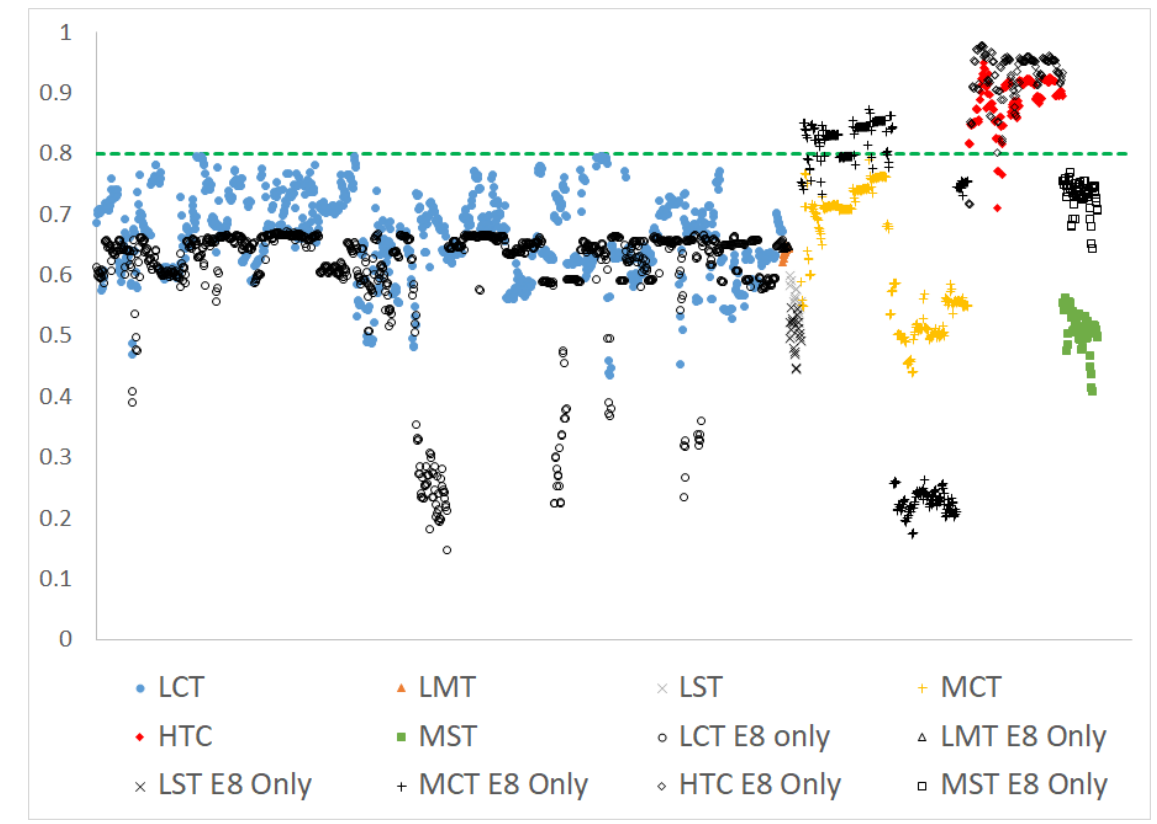

Figure 15. Applicability of various critical experiments for validation of PWR spent fuel storage system using SCALE 6.2 and ENDF/B-VIII.0 covariance libraries. 


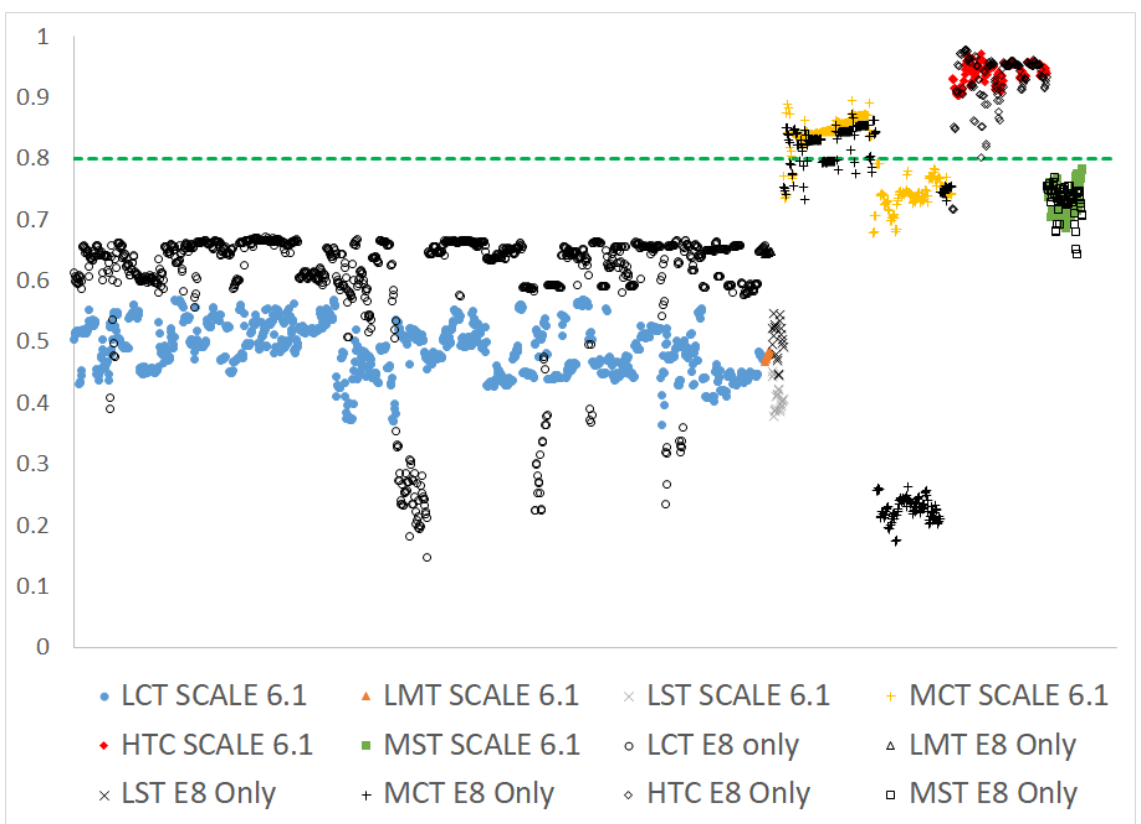

Figure 16. Applicability of various critical experiments for validation of PWR spent fuel storage system using SCALE 6.1 and ENDF/B-VIII.0 covariance libraries.

\section{ENDF/B-VIII.0 COVARIANCE ASSESSMENT FOR ADVANCED REACTOR APPLICATIONS}

Sensitivity data have been generated for a number of potential advanced reactor systems [1]. These sensitivity data are used in this section to investigate the impact of ENDF/B-VIII.0 covariance data on data induced uncertainty for these systems. The approach is analogous to the analysis of data induced uncertainties in critical experiments presented in Section 3.2. The advanced reactor systems analyzed include gas cooled, graphite moderated systems, molten salt reactors, and sodium-cooled fast reactors.

\subsection{GAS COOLED REACTORS}

To consider both graphite-moderated gas-cooled reactor systems, the HTR 10 [14] pebble-bed reactor and the prismatic HTTR [15] were modeled. HTR-10 is a small $10 \mathrm{MW}_{\text {th }}$ prototype pebble-bed reactor at Tsinghua University in China [14]. For the initial critical configuration of HTR-10, the cylindrical core consisted of a mixture of 9,627 fuel pebbles and 7,263 dummy pebbles at a packing fraction of $61 \%$, that are surrounded by graphite reflector structures. The fuel pebbles have a $2.5 \mathrm{~cm}$ radius inner fuel zone that is surrounded by a $0.5 \mathrm{~cm}$ thick graphite layer. The fuel zone contains about 8,000 TRISO particles distributed within a graphite matrix. The TRISO particles contain a fuel kernel consisting of uranium dioxide with $17 \mathrm{wt} . \%$ enrichment. The HTTR is a graphite-moderated helium-cooled $30 \mathrm{MW}_{\text {th }}$ prismatic reactor built by the Japan Atomic Energy Agency. The core of the HTTR consists of hexagonal graphite blocks that are stacked vertically into columns. The fuel blocks contain fuel compacts that are composed of TRISO fuel particles dispersed in a graphite matrix with a volumetric packing fraction of $30 \%$. The TRISO particles contain a fuel kernel consisting of uranium dioxide with enrichments ranging between 3.4 and $9.9 \mathrm{wt} . \%$. CSAS/KENO-VI models prepared for earlier studies of the HTR-10 and HTTR with SCALE [16-18] were used for the present work. 
As discussed in Section 3.2.1, the ENDF/B-VIII.0 covariance data for graphite cannot be used correctly in currently available versions of SCALE, but will be fully functional with SCALE 6.3, which will include ENDF/B-VIII.0 data libraries. This leads to an unrealistic increase in the data induced uncertainty due to the $40 \%$ uncertainty used to patch this gap in the covariance data. Aside from the artificial increase caused by graphite, the uncertainty contributed by ${ }^{235} \mathrm{U}$ nu-bar increases for both reactor systems from approximately $0.37 \%$ to approximately $0.46 \%$. The uncertainty from ${ }^{235} \mathrm{U}$ fission also increases in ENDF/B-VIII.0 for both systems. The inelastic scattering reaction in ${ }^{238} \mathrm{U}$ actually contributes less uncertainty using the ENDF/B-VIII.0 library than the SCALE 6.2 library.

Excluding all graphite reactions, the data induced uncertainty in the HTR-10 system is $0.53 \%$ with the SCALE 6.2 data and $0.50 \%$ with the ENDF/B-VIII.0 covariance data. The uncertainty from several reactions, including ${ }^{235} \mathrm{U} \chi$ and ${ }^{235} \mathrm{U}$ scattering reactions, is significantly lower with the ENDF/B-VIII.0 data. The case is reversed for the HTTR model, for which the SCALE 6.2 library data induced uncertainty, without graphite reactions, is $0.54 \%$. The uncertainty increases to $0.60 \%$ with the ENDF/BVIII.0 library. This increase appears to be primarily the result of the increase in the ${ }^{235} \mathrm{U}$ nu-bar uncertainty.

\subsection{MOLTEN SALT REACTORS}

Three different molten salt reactor unit cell models are considered: (1) an infinite medium representative for a fast-spectrum MSR concept, based on $\mathrm{NaCl}$ as the carrier salt, (2) a graphite-moderated unit cell for which a fuel salt channel is surrounded by the graphite moderator, and (2) a zirconium-hydride moderated unit cells with fuel salt surrounding a small-diameter moderator rod. All three systems are considered both fresh and with depleted fuel. All MSR models are taken from earlier studies at ORNL [19-24].

The data induced uncertainty in the fast chloride system with fresh fuel drops from $1.91 \%$ with the SCALE 6.2 data to $1.49 \%$ with the ENDF/B-VIII.0 data. Similarly, the model with depleted fuel experiences a decrease from $1.98 \%$ to $1.48 \%$. Both cases see a dramatic increase in the uncertainty in $k_{\text {eff }}$ due to the ${ }^{239} \mathrm{Pu}$ reaction, but this is overwhelmed by the significant drop in the ${ }^{238} \mathrm{U}$ inelastic scattering uncertainty. The energy dependent uncertainty profile for this reaction is shown in Figure 12. This reaction contributes an uncertainty of over $1.5 \%$ with the SCALE 6.2 library, in both cases, but less than $0.5 \%$ with the ENDF/B-VIII.0 covariance data.

The thermal unit cell model with fresh fuel moderated by graphite has similar overall data induced uncertainty excluding all graphite reactions. The uncertainty resulting from the SCALE 6.2 data is $0.54 \%$, and with the ENDF/B-VIII.0 data it is $0.52 \%$. The top two uncertainty contributors from both libraries are ${ }^{235} \mathrm{U}$ nu-bar and ${ }^{238} \mathrm{U}$ inelastic scattering. The ${ }^{235} \mathrm{U}$ nu-bar uncertainty increases in ENDF/B-VIII.0, but the uncertainty contribution from ${ }^{238} \mathrm{U}$ inelastic scattering drops. The unit cell model with depleted fuel experiences a significant increase in data induced uncertainty with the ENDF/B-VIII.0 library compared to the SCALE 6.2 library. The top contributor from the SCALE 6.2 data is ${ }^{238} \mathrm{U}$ inelastic scattering, and it drops from $0.35 \%$ to $0.22 \%$ with the ENDF/B-VIII.0 data. The uncertainty in ${ }^{239} \mathrm{Pu}$ capture and fission both increase significantly in ENDF/B-VIII.0, resulting in the observed overall increase in the data induced uncertainty.

The thermal unit cell model with fresh fuel moderated by zirconium hydride has lower data induced uncertainty with the ENDF/B-VIII.0 covariance data than with the SCALE 6.2 data. As with the graphite moderated cell, the primary differences are in ${ }^{235} \mathrm{U}$ nu-bar and ${ }^{238} \mathrm{U}$ inelastic scattering. In this case, though, the decrease in the ${ }^{238} \mathrm{U}$ inelastic scattering outweighs in the increase in ${ }^{235} \mathrm{U}$ nu-bar. For the model with depleted fuel, the data induced uncertainty increases from $0.66 \%$ with the SCALE 6.2 data to $0.99 \%$ with the ENDF/B-VIII.0 data. The primary differences are increases in the ${ }^{239} \mathrm{Pu}$ fission and capture reactions. The top two reactions contributing to uncertainty in the SCALE 6.2 data are capture in ${ }^{91} \mathrm{Zr}$ and ${ }^{92} \mathrm{Zr}$. The uncertainty contribution of these reactions does not change in the ENDF/B-VIII.0 data.

In summary, the changes introduced in the data induced uncertainty in $k_{\text {eff }}$ in the ENDF/B-VIII.0 covariance set vary depending on the molten salt model. There are currently a wide range of molten salt 
reactor concepts under investigation, so it is difficult to narrow this evaluation from the broad set of systems and a simple unit cell model.

\subsection{SODIUM FAST REACTORS}

Two sodium fast reactor (SFR) models are considered here: a fuel assembly of a medium-sized metallic fuel core (MET1000) and a fuel assembly of a large oxide core (MOX3600) as defined in the subexercises of the OECD/NEA Benchmark for Uncertainty Analysis in Modeling of SFRs [25, 26]. The MOX3600 fuel pins contain annular uranium-transuranic (U-TRU) oxide, and the MET1000 pins contain cylindrical U-TRU-Zr metal fuel. In both cases, the fuel is end-of-equilibrium-cycle (EOEC) fuel.

The data induced uncertainty in the MET1000 model decreases from 1.40\% with the SCALE 6.2 covariance data to $1.07 \%$ with the ENDF/B-VIII.0 data. The uncertainty contribution from ${ }^{238} \mathrm{U}$ inelastic scattering is $1.17 \%$ using the SCALE 6.2 data but only $0.49 \%$ based on the ENDF/B-VIII.0 covariance data. This change more than offsets the increase in ${ }^{239} \mathrm{Pu}$ fission from $0.20 \%$ to $0.56 \%$. The largest contribution from ${ }^{23} \mathrm{Na}$ is elastic scattering in both libraries and contributes $0.33 \%$ to the data induced uncertainty in both cases.

Similar changes impact the MOX3600 model. The ${ }^{239} \mathrm{Pu}$ fission uncertainty increases from $0.20 \%$ based on the SCALE 6.2 data to $0.57 \%$ with the ENDF/B-VIII.0 data. The contribution from ${ }^{238} \mathrm{U}$ inelastic scatter drops from $1.33 \%$ to $0.34 \%$. The huge drop in uncertainty, shown in Figure 12, has significant impact on several of the systems discussed in this report. The MOX3600 model is just one more example, and further evidence that changes in the covariance data can have significant effects on the predicted uncertainty of calculated $k_{\text {eff }}$ values.

\section{AUGMENTED ENDF/B-VIII.0 COVARIANCE DEVELOPMENT}

\subsection{THE APPROACH TO A FIRST AUGMENTED COVARIANCE MATRIX}

The goal of this work to build up an augmented ENDF/B-VIII.0 covariance matrix that will incorporate the additional knowledge gained about particular combinations of nuclear data quantities from including feedback from integral experiments. The augmented covariance matrix should preserve the uncertainties on the individual nuclear data quantities representative of what can be expected from state-of-the-art differential measurements as would be used in reaction rate calculations while at the same time reflecting that nuclear data in particular functional combinations have been tuned to reproduce the expected $\mathrm{k}_{\text {eff }}$ values of certain benchmark uncertainties with low uncertainties.

An iterative approach is taken to accomplishing this task. It is hoped that through small incremental augmentations with frequent checkpointing, a better end result can be achieved in increased understanding relative to single absolute augmentation to the ENDF/B-VIII.0 covariance matrix. Therefore, the goal of the first augmented file is to move in the right direction of achieving the goals laid out above rather than provide a complete solution.

With this in mind, the first augmentation to the ENDF/B-VIII.0 covariance matrix described in this section is intended to only estimate cross-correlation matrices which are not already present in ENDF/BVIII.0. Namely, the fission-nu-bar correlations will be estimated for ${ }^{239} \mathrm{Pu},{ }^{235} \mathrm{U}$ and ${ }^{238} \mathrm{U}$. These crosscorrelations do not exist in ENDF/B-VIII.0 library as it is argued that the differential experimental techniques used to measure the fission cross section and nu-bar are independent. Furthermore, different physics models are used to evaluate each quantity. However, the cross correlations between these two quantities are introduced when tuning the combined set of nuclear data to match integral measurements of $\mathrm{k}_{\text {eff. }}$

Only the cross-correlations, not the covariance, between fission-nu-bar correlations for ${ }^{239} \mathrm{Pu},{ }^{235} \mathrm{U}$ and

${ }^{238} \mathrm{U}$ will be augmented. It is understood that this first step in augmentation will create a mismatched covariance file where existing cross-correlations between the fission and capture reactions will be 
retained based on evaluation of differential data, while the fission-nu-bar correlations will be those which come from the incorporation of the integral benchmarks. However, in the multi-step process the fissioncapture cross correlations will be updated eventually as well to incorporate the knowledge gained from the integral benchmarks. The missing fission-nu-bar correlations are the biggest missing piece. Once again, it is important to be clear that not all of the improved predictive power will be realized only through the cross-correlations between fission and nu-bar. Rather the goal is to estimate the true correlation.

The multi-step approach to augmenting the ENDF/B-VIII.0 covariance matrix will also be done in a multiresolution fashion. The covariance matrices for nuclear data have an energy component, usually described by an energy group structure. The goal is to first determine the bulk, or coarse-group, correlations and then to refine the energy fidelity of the covariance matrices. This will ensure that the bulk behavior is captured correctly before all of the details are fine tuned.

For the first demonstration, a three-group structure was selected,
1. Fast group
$20 \mathrm{MeV}-50 \mathrm{keV}$
2. Intermediate group
$50 \mathrm{keV} \quad-0.625 \mathrm{eV}$
3. Thermal group
$0.625 \mathrm{eV}-10^{-5} \mathrm{eV}$

The value of $50 \mathrm{keV}$ was chosen as opposed to the traditional value of $100 \mathrm{keV}$ used by the ICSBEP to separate the fast group from the intermediate group to match on to the nearest energy boundary of the 56group structure of the SCALE multi-group covariance library.

\subsection{THE RESULTS OF AN AUGMENTED COVARIANCE MATRIX}

To generate the first augmented ENDF/B-VIII.0 covariance matrix the TSURFER module of the SCALE code system was used to systematically determine the missing cross correlations and the AMPX module was used to populate the cross-correlations matrices. The TSURFER code uses the generalized linear least-squares method to consolidate a prior set of measured integral responses such as $k_{\text {eff }}$ or reaction rates and the corresponding calculated values obtained using the SCALE nuclear analysis code system. The initial estimates for the computed and measured responses are improved by adjusting the experimental values and the nuclear data used in the transport calculations, considering their correlated uncertainties, so that the most self-consistent set of data is obtained. Consolidation of the original integral experiment data and calculated results reduces the prior uncertainty in the response estimates because additional information has been incorporated.

In this study TSURFER was used to mimic the adjustment process of the integral data validation feedback loop on the covariance side. In the TSURFER analysis a full adjustment was done based on the available benchmark sensitivity profiles in the VALID suite of 600 benchmark experiments from the ICSBEP and other sources. The adjustment was done to all of the nuclear data for the isotopes appearing in the benchmarks, however, as described above, only the posterior cross-correlation matrices were extracted. This choice was made to preserve the values of the uncertainties on the individual nuclear data quantities to correspond to uncertainty in differential measurements, while, at the same time, the added cross-correlations will reflect the knowledge of the combinations of fission and nu-bar in three different energy regions; fast, intermediate and thermal.

The coarse group correlation coefficients for the fast-fission to fast-nu-bar and intermediate-fission to fast-nu-bar were assessed by evaluating the TSURFER GLLS adjustment based on three different groups of benchmarks in VALID. The adjustment of twelve PU-MET-FAST cases resulted in values of -53\%, $33 \%$ correlations respectively. While the adjustment based on two MIX-COMP-FAST cases gave -18\% and $-19 \%$. Lastly, the adjustments made considering all of the benchmarks containing plutonium in the VALID library independent of the fuel form or spectrum confirmed a strong negative correlation between fission and nu-bar. The values were found to be $-54 \%$ and $-34 \%$ respectively, which match intuition in approximate magnitude and sign. 
The estimates of the cross-correlations between fission and thermal and intermediate nu-bar were consistently estimated by TSURFER based on multiple different combinations of VALID benchmarks included in the analysis. The replicability of the sign and rough magnitude of the cross-correlations regardless of the choice integral benchmarks supports strengthens the confidence in the selected approach.

Table 2. Coarse group cross correlation estimates for ${ }^{239} \mathrm{Pu}$ based on including different VALID benchmarks in the TSURFER GLLS adjustment (in percent).

\begin{tabular}{|c|c|c|c|}
\hline \multicolumn{4}{|c|}{ PU-MET-FAST (PMF) } \\
\hline & fission fast & fission intermediate & fission thermal \\
\hline nu-bar fast & -53 & -33 & \\
\hline nu-bar intermediate & -2 & -1 & -1 \\
\hline nu-bar thermal & N/A & $\mathrm{N} / \mathrm{A}$ & $\mathrm{N} / \mathrm{A}$ \\
\hline \multicolumn{4}{|c|}{ PU-SOL-THERM (PST) (single experiment from each benchmark series) } \\
\hline & fission fast & fission intermediate & fission thermal \\
\hline nu-bar fast & $\mathrm{N} / \mathrm{A}$ & $\mathrm{N} / \mathrm{A}$ & N/A \\
\hline nu-bar intermediate & -1 & -2 & -2 \\
\hline nu-bar thermal & -7 & -10 & -10 \\
\hline \multicolumn{4}{|c|}{ PU-SOL-THERM (PST) (all experiments) } \\
\hline & fission fast & fission intermediate & fission thermal \\
\hline nu-bar fast & $\mathrm{N} / \mathrm{A}$ & N/A & N/A \\
\hline nu-bar intermediate & -2 & -3 & -3 \\
\hline nu-bar thermal & -9 & -15 & -16 \\
\hline \multicolumn{4}{|c|}{ MIX-COMP-FAST (MCF) } \\
\hline & fission fast & fission intermediate & fission thermal \\
\hline nu-bar fast & -18 & -19 & \\
\hline nu-bar intermediate & -4 & -5 & -5 \\
\hline nu-bar thermal & N/A & N/A & N/A \\
\hline \multicolumn{4}{|c|}{ MIX-SOL-THERM (MST) (single experiment from each benchmark series) } \\
\hline & fission fast & fission intermediate & fission thermal \\
\hline nu-bar fast & N/A & N/A & N/A \\
\hline nu-bar intermediate & -2 & -3 & -3 \\
\hline nu-bar thermal & -8 & -13 & -14 \\
\hline
\end{tabular}




\begin{tabular}{|c|c|c|c|}
\hline \multicolumn{4}{|c|}{ MIX-SOL-THERM (MST) (all experiments) } \\
\hline & fission fast & fission intermediate & fission thermal \\
\hline nu-bar fast & N/A & N/A & N/A \\
\hline nu-bar intermediate & -3 & -4 & -4 \\
\hline nu-bar thermal & -11 & -17 & -18 \\
\hline \multicolumn{4}{|c|}{ MIX-COMP-THERM (MCT) (single experiment from each benchmark series) } \\
\hline & fission fast & fission intermediate & fission thermal \\
\hline nu-bar fast & $\mathrm{N} / \mathrm{A}$ & N/A & N/A \\
\hline nu-bar intermediate & -3 & -5 & -5 \\
\hline nu-bar thermal & -4 & -7 & -7 \\
\hline \multicolumn{4}{|c|}{ MIX-COMP-THERM (MCT) (all experiments) } \\
\hline & fission fast & fission intermediate & fission thermal \\
\hline nu-bar fast & N/A & N/A & N/A \\
\hline nu-bar intermediate & -4 & -5 & -5 \\
\hline nu-bar thermal & -6 & -10 & -11 \\
\hline \multicolumn{4}{|c|}{ All cases in VALID containing plutonium } \\
\hline & fission fast & fission intermediate & fission thermal \\
\hline nu-bar fast & -54 & -34 & \\
\hline nu-bar intermediate & -3 & -3 & -3 \\
\hline nu-bar thermal & -7 & -10 & -10 \\
\hline
\end{tabular}

The cross-correlations between fission and nu-bar for ${ }^{235} \mathrm{U}$ were estimated using the same procedure as for plutonium, that is by analyzing multiple different combinations of integral benchmarks from VALID included in the TSURFER calculations. The TSURFER calculations were done by considering uraniumcontaining benchmarks from each ICSBEP category and including only a single case from each experimental series or all of the cases as well as combining all of the uranium-containing cases in VALID into a single TSURFER calculation. The results consistently produced negative coarse group crosscorrelation coefficients of magnitude between $8-50 \%$. Table 3 presents two representative cases for the determination of the cross-correlation coefficients for ${ }^{235} \mathrm{U}$. 
Table 3. Coarse group cross correlation estimates for ${ }^{235} \mathbf{U}$ based on including different VALID benchmarks in the TSURFER GLLS adjustment (in percent).

INTER-MET-FAST (IMF) (single experiment from each benchmark series)

\begin{tabular}{lccc}
\hline & fission fast & fission intermediate & fission thermal \\
\cline { 2 - 4 } nu-bar fast & -46 & -33 & -37 \\
nu-bar intermediate & -28 & -20 & -23 \\
nu-bar thermal & -38 & -26 & -30 \\
\hline
\end{tabular}

INTER-MET-FAST (IMF) (all experiments)

\begin{tabular}{lccc}
\hline & fission fast & fission intermediate & fission thermal \\
\cline { 2 - 4 } nu-bar fast & -46 & -34 & -38 \\
nu-bar intermediate & -28 & -20 & -23 \\
nu-bar thermal & -39 & -27 & -31 \\
\hline LEU-COMP-THERM (LCT) (single experiment from each benchmark series) & fission thermal \\
\hline & fission fast & fission intermediate & -13 \\
nu-bar fast & -10 & -8 & -32 \\
nu-bar intermediate & -24 & -17 & -36 \\
nu-bar thermal & -23 & -13 & fission thermal \\
\hline LEU-COMP-THERM (LCT) (all experiments) & fission fast & & -11 \\
\hline & -9 & -9 & -30 \\
nu-bar fast & -23 & -18 & -36 \\
nu-bar intermediate & -23 & -13 & \\
nu-bar thermal & & fission intermediate & \\
\hline
\end{tabular}

Lastly, the ${ }^{238} \mathrm{U}$ cross-correlation coefficients between fast fission and fast nu-bar were estimated using the same approach with results shown in Table 4. 
Table 4. Coarse group cross correlation estimates for ${ }^{238} \mathrm{U}$ fast fission to fast nu-bar based on including different VALID benchmarks in the TSURFER GLLS adjustment (in percent).

HEU-MET-FAST (HMF) (single experiment from each benchmark series)

\begin{tabular}{|c|c|c|c|}
\hline & fission fast & fission intermediate & fission thermal \\
\hline nu-bar fast & -5 & & \\
\hline \multicolumn{4}{|c|}{ nu-bar intermediate } \\
\hline \multicolumn{4}{|l|}{ nu-bar thermal } \\
\hline \multicolumn{4}{|c|}{ HEU-MET-FAST (HMF) (all experiments) } \\
\hline & fission fast & fission intermediate & fission thermal \\
\hline nu-bar fast & -5 & & \\
\hline \multicolumn{4}{|c|}{ nu-bar intermediate } \\
\hline nu-bar thermal & & & \\
\hline
\end{tabular}

INTER-MET-FAST (IMF) (single experiment from each benchmark series)

\begin{tabular}{lcc} 
& fission fast & fission intermediate \\
\cline { 2 - 3 } nu-bar fast & -5 & fission thermal \\
nu-bar intermediate & & \\
nu-bar thermal & & \\
\hline
\end{tabular}

INTER-MET-FAST (IMF) (all experiments)

\begin{tabular}{|c|c|c|c|}
\hline & fission fast & fission intermediate & fission thermal \\
\hline nu-bar fast & -5 & & \\
\hline \multicolumn{4}{|c|}{ nu-bar intermediate } \\
\hline nu-bar thermal & & & \\
\hline
\end{tabular}

LEU-COMP-THERM (LCT) (single experiment from each benchmark series)

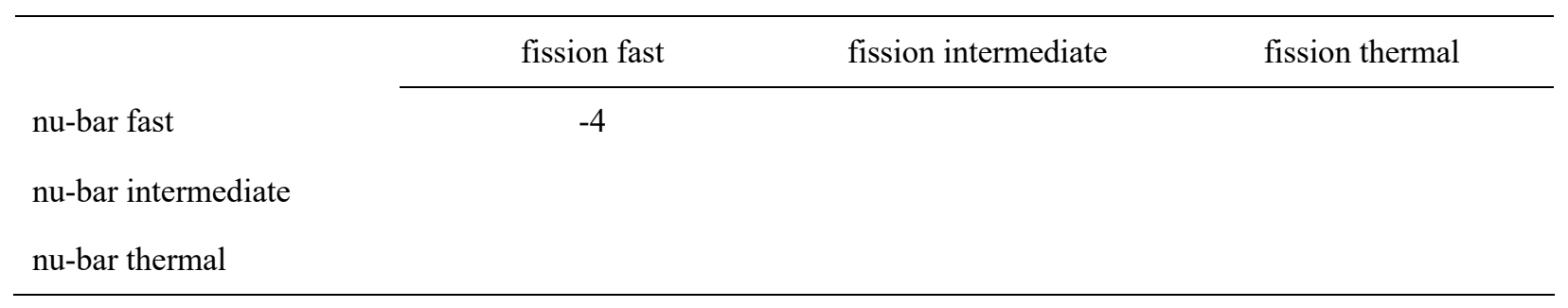


LEU-COMP-THERM (LCT) (all experiments)

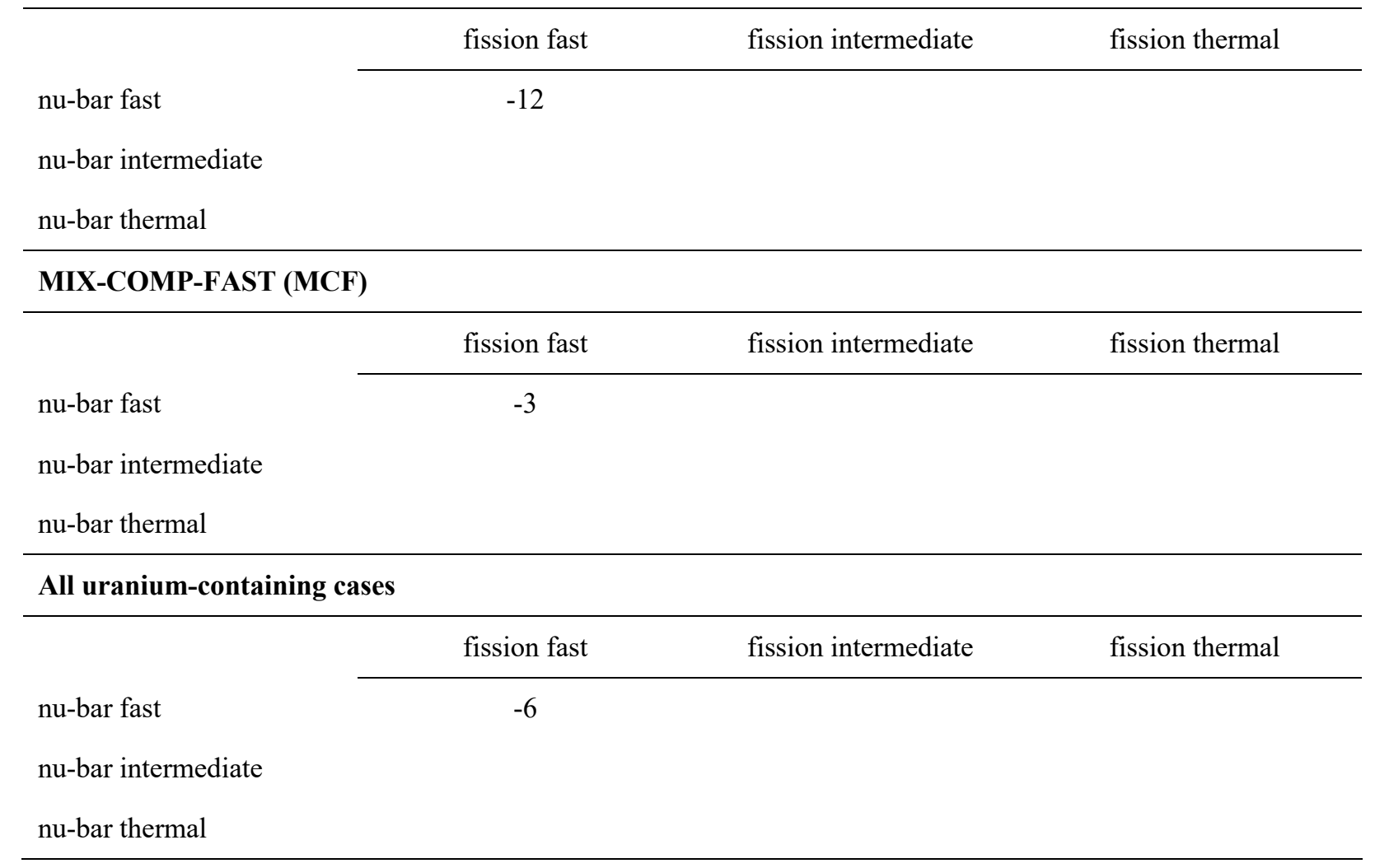

The augmented covariance matrix based on this approach for ENDF/B-VIII.0 is estimated as presented in Table 5.

Table 5. Estimations of the coarse group cross-correlations for the first augmented ENDF/B-VIII.0 library (in percent).

\begin{tabular}{lccc}
\hline${ }^{239} \mathbf{P u}$ & & \\
\hline & fission fast & fission intermediate & fission thermal \\
\cline { 2 - 4 } nu-bar fast & -53 & -33 & \\
nu-bar intermediate & -1 & -2 & -2 \\
nu-bar thermal & -7 & -10 & -11 \\
\hline${ }^{235} \mathbf{U}$ & & fission intermediate & fission thermal \\
\hline & fission fast & -33 & -13 \\
nu-bar fast & -46 & -20 & -32 \\
nu-bar intermediate & -28 & -26 & -33 \\
nu-bar thermal & -38 & & \\
\hline
\end{tabular}




\begin{tabular}{lccc}
\hline${ }^{238} \mathbf{U}$ & & & \\
\hline & fission fast & fission intermediate & fission thermal \\
\cline { 2 - 3 } & -5 & & \\
nu-bar fast & & & \\
nu-bar intermediate & & \\
\hline
\end{tabular}

\section{AUGMENTED ENDF/B-VIII.0 COVARIANCE MATRIX RESULTS FOR INTEGRAL EXPERIMENTS}

\subsection{HEU-MET-FAST SYSTEMS}

The impact of using the augmented ENDF/B-VIII.0 covariance library is visually evident from Figure 17. For many of the benchmarks the augmented covariance library results in a noticeably smaller amount of propagated uncertainty. For many cases the reduction in uncertainty is on the order of $10 \%$, which is a good improvement given that only a small subset of cross-correlation matrices was estimated. In the extreme peaks of the propagated uncertainty, almost no change is noticeable as those cases are dominated by uncertainty from other isotopes than ${ }^{235} \mathrm{U}$ and ${ }^{238} \mathrm{U}$, the covariance for which was not affected in the first iteration. Section 6.5 will present a quantified summary of these results and it is important to point out here, the four of the benchmarks on the right side of the figure fall within the nuclear data uncertainty error bars from ENDF/B-VIII.0 covariance while are outside of the one-standard-deviation error bars of the augmented library.

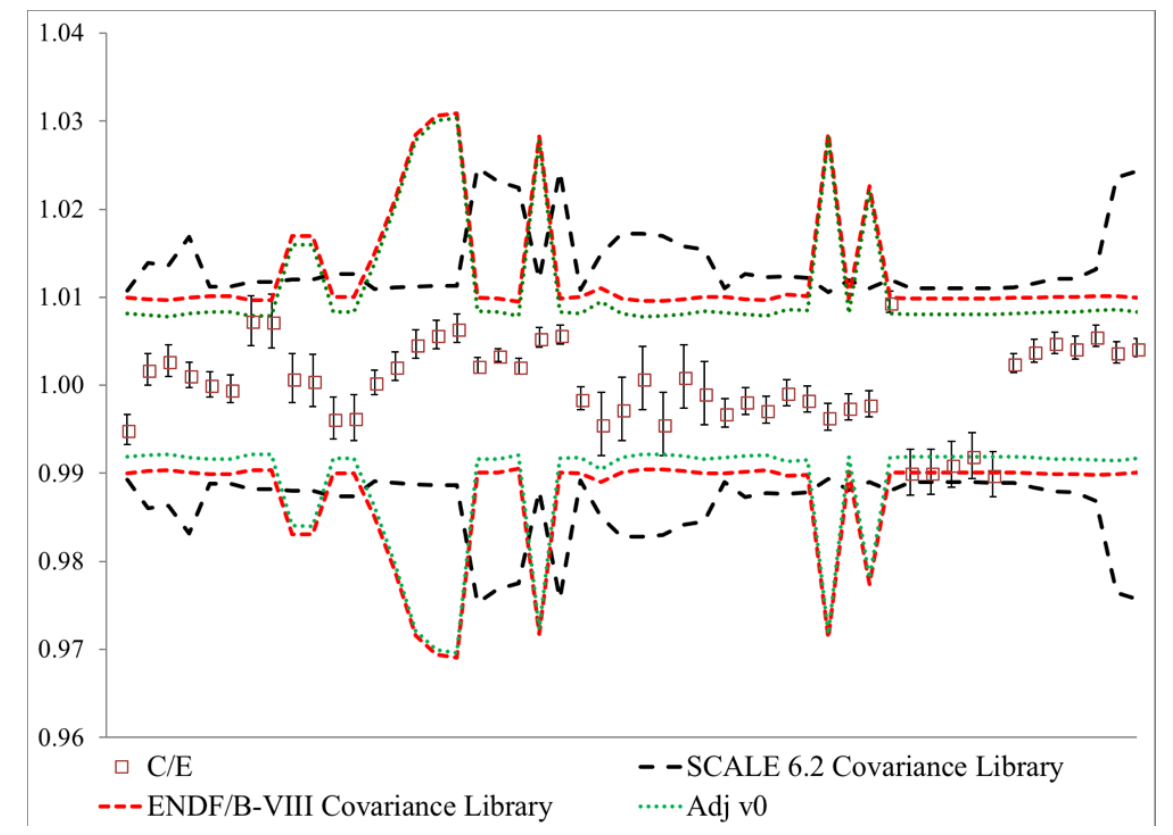

Figure 17. Results for HEU-MET-FAST systems. The experiments are sorted by their ICBSEP-assigned number on the horizontal axis. $\mathrm{C} / \mathrm{E}$ values are shown on the vertical axis. 


\subsection{LEU-COMP-THERM SYSTEMS}

In Figure 18, the augmented covariance library reduces the amount of propagated uncertainty in the same manner as was observed in Figure 17. While the first iteration of the augmented covariance library does not eliminate the gap between $\mathrm{C} / \mathrm{E}$ discrepancy and the propagated nuclear data uncertainty, it does show the methodology of introducing cross-correlations does move the nuclear data error bands in the right direction. As most of these benchmarks are rodded lattice configurations moderated by water, introducing further cross-correlations between hydrogen and ${ }^{235} \mathrm{U}$ and ${ }^{238} \mathrm{U}$ should reduce the size of the nuclear data error bands.

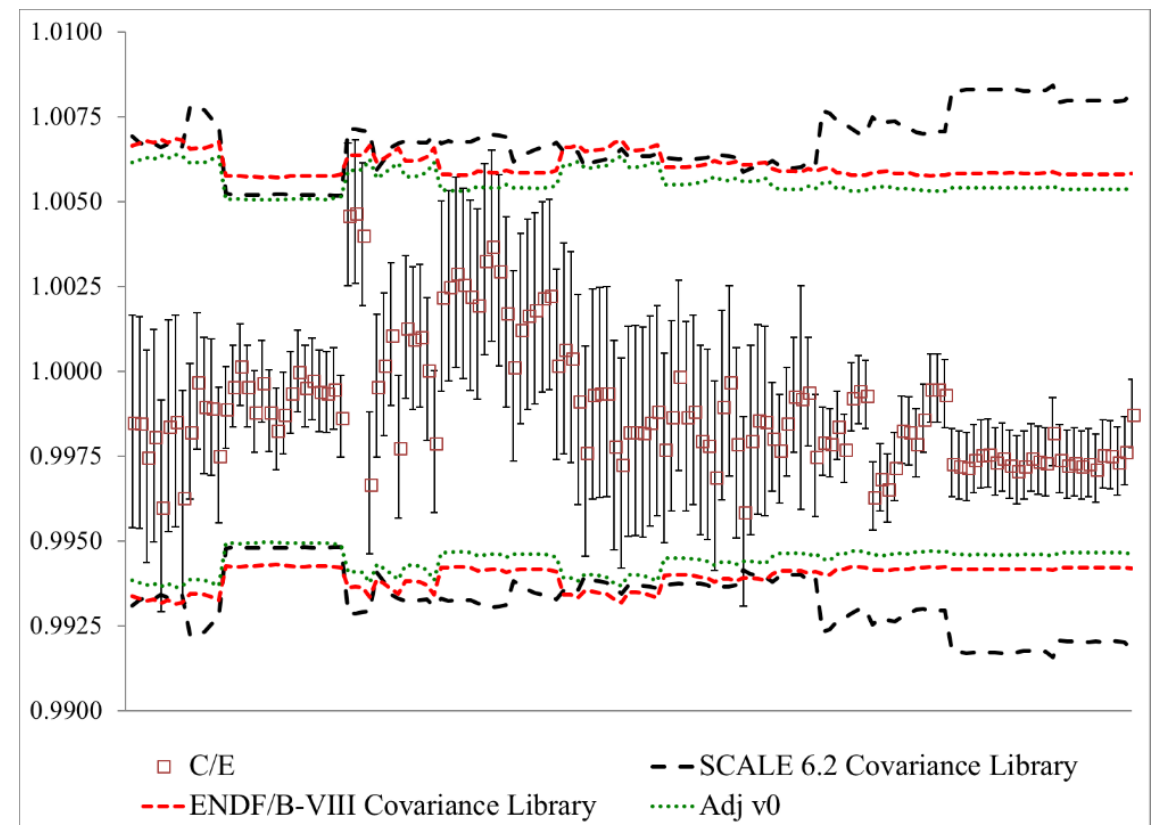

Figure 18. Results for LEU-COMP-THERM systems. The experiments are sorted by their ICBSEP-assigned number on the horizontal axis. $\mathrm{C} / \mathrm{E}$ values are shown on the vertical axis.

\subsection{MIX-COMP-THERM SYSTEMS}

The augmented covariance library did not have a significant impact on the MCT set of integral benchmarks. In fact, as will be reported in Section 3.2.5 in Table 6, the average uncertainty due to nuclear data only went down to $958 \mathrm{pcm}$ from $973 \mathrm{pcm}$, which is not a significant improvement. For future iterations of the augmented covariance library, the MCT systems will be an important check, as even a slight reduction in uncertainty may put many of the integral benchmarks on the right side of the plot outside of one standard deviation error bars. It is important to keep in mind that the goal of the augmented covariance library is to reduce the discrepancy between the calculated and measured integral values while staying on the conservative side of estimating the uncertainty in nuclear data. These systems will be important for estimating the cross-correlations between ${ }^{239} \mathrm{Pu}$ and ${ }^{235} \mathrm{U}$ and ${ }^{238} \mathrm{U}$. 


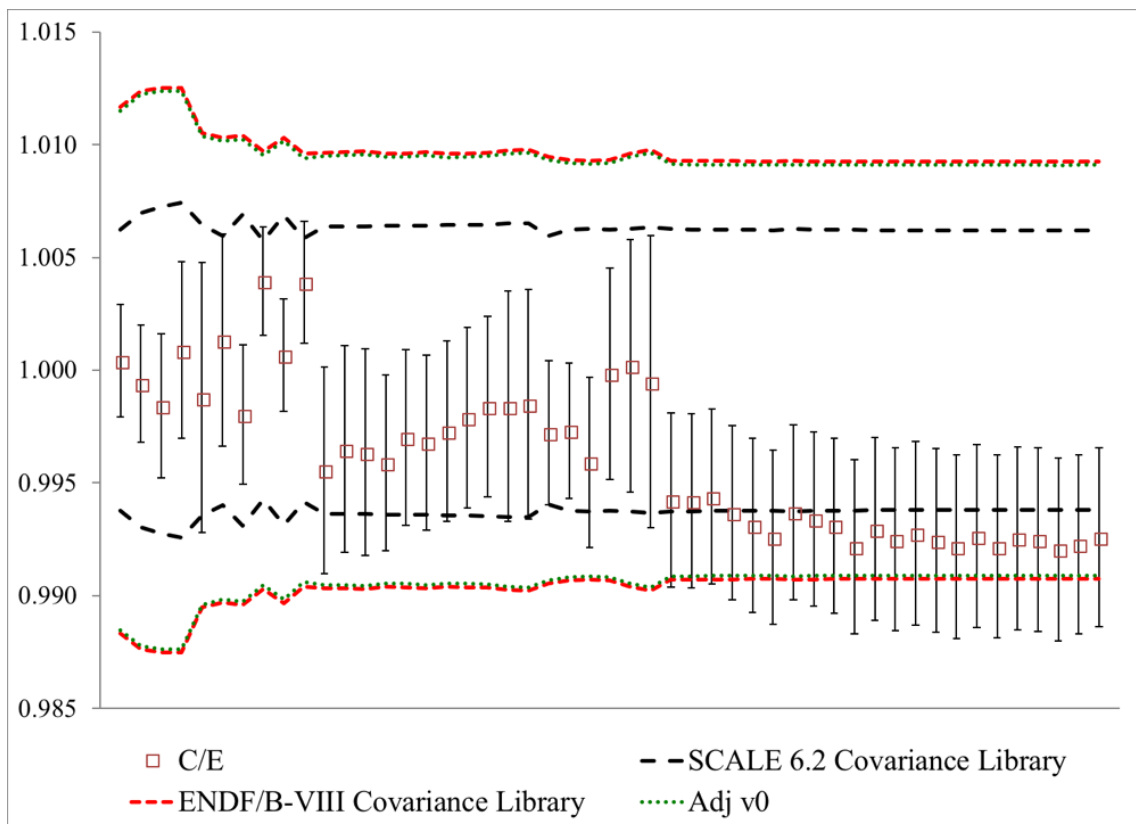

Figure 19. Results for the MIX-COMP-THERM systems. The experiments are sorted by their ICBSEPassigned number on the horizontal axis. $\mathrm{C} / \mathrm{E}$ values are shown on the vertical axis.

\subsection{PU-MET-FAST SYSTEMS}

For the PMF systems the augmented covariance library, namely the estimated cross-correlations between fission and nu-bar for ${ }^{239} \mathrm{Pu}$ have reduced the difference between ENDF/B-VIII.0 and SCALE 6.2 covariance libraries approximately by a factor of 2 . It may be difficult to reduce the amount of nuclear data uncertainty any further for the PMF systems keeping in the spirit of the methodology laid out in Section 2. A detailed analysis of the distribution of the remaining uncertainty will be necessary. Further, the nuclear data covariance is shared among all of the other integral systems using plutonium, therefore, efforts to reduce the propagated uncertainty in the PMF systems will also impact the other benchmark categories. 


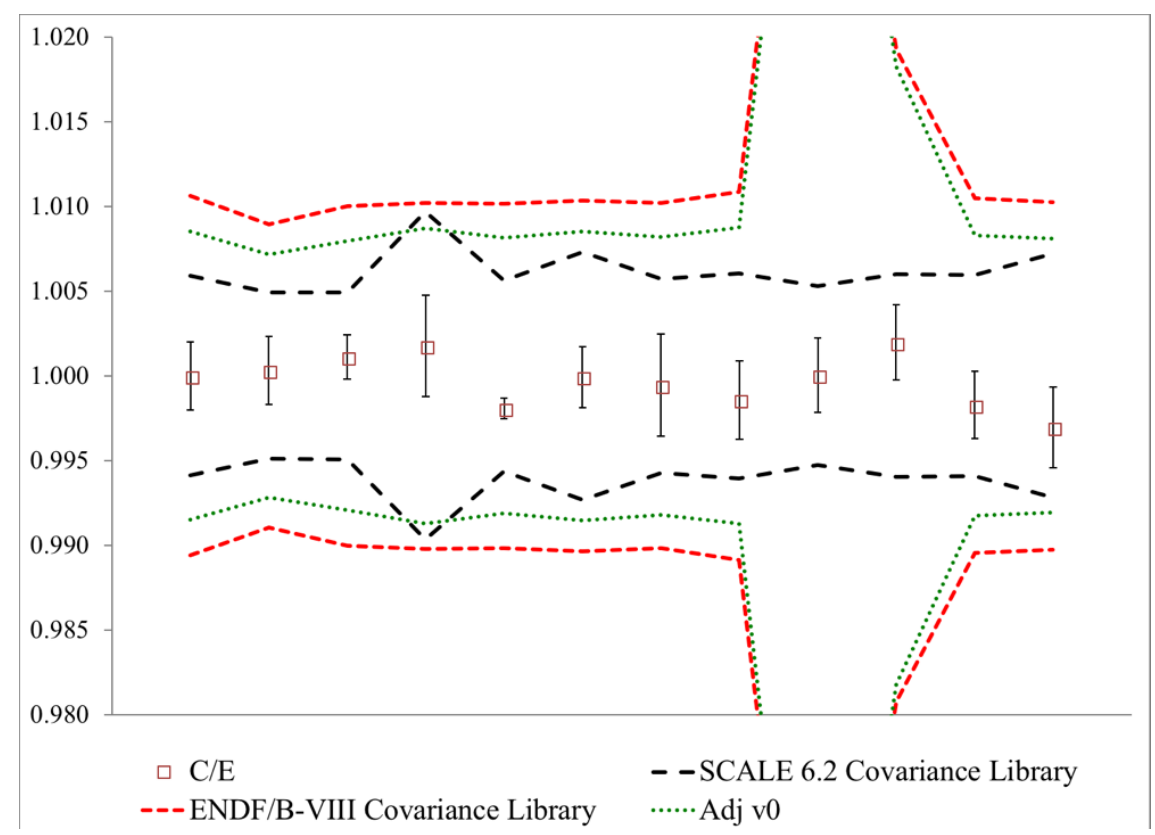

Figure 20. Results for PU-MET-FAST systems. The experiments are sorted by their ICBSEP-assigned number on the horizontal axis. $C / E$ values are shown on the vertical axis.

\subsection{SUMMARY OF ALL CATEGORIES}

From Table 5, a summary evaluation can be made of the performance of the ENDF/B-VIII.0 augmented library in this first iteration. The augmented library has achieved the desired result. As expected, the augmented library has reduced the average amount of uncertainty propagated form nuclear data universally across all 10 benchmark experiment categories in VALID. The right-most column in Table 5 shows that the reduction in uncertainty propagated from the augmented ENDF/B-VIII.0 covariance file improves the percentage of cases within one standard deviation only for three of the experiment categories. This is not unexpected as the first iteration of the augmented library has only estimated a small portion, though important portion, of the cross-correlations for the entire library. Therefore, it was reasonable to expect that perfect agreement will not be reached with this iteration. On the other hand, there is a clear improvement in reducing the amount of uncertainty propagated from nuclear data even with a small subset of cross-correlations. In conclusion the first iteration of the augmented library has demonstrated that the chosen path forward is in the right direction of achieving agreement between the discrepancies in calculated and experimentally measured integral benchmarks and the propagated uncertainty from nuclear data. 
Table 6. Uncertainty Summary for Ten Categories of Experiments in VALID

(Uncertainties are reported in pcm).

\begin{tabular}{ccccccccc}
\hline Category & $\begin{array}{c}\text { Avg. Exp. } \\
\text { Unc. }\end{array}$ & $\begin{array}{c}\text { St Dev of } \\
\text { C/E Values }\end{array}$ & $\begin{array}{c}\text { SCALE 6.2 } \\
\text { XS Unc }\end{array}$ & $\begin{array}{c}\text { ENDF/B8 } \\
\text { XS Unc }\end{array}$ & $\begin{array}{c}\text { ENDF/B8 } \\
\text { Augmented } \\
\text { XS Unc }\end{array}$ & $\begin{array}{c}\text { \% Cases wrt } \\
\text { Exp Unc }\end{array}$ & $\begin{array}{c}\text { \% Cases wrt } \\
\text { ENDF/B8 } \\
\text { XS Unc }\end{array}$ & $\begin{array}{c}\text { \% Cases wrt } \\
\text { Aug. E8 } \\
\text { XS Unc }\end{array}$ \\
\hline HMF & 193 & 476 & 1,380 & 1,442 & 1121 & 50.0 & 98 & 90 \\
HST & 494 & 593 & 1,051 & 655 & 611 & 42.3 & 75 & 75 \\
IMF & 269 & 359 & 1,522 & 1,593 & 1496 & 30.8 & 100 & 100 \\
LCT & 195 & 167 & 677 & 606 & 557 & 58.6 & 100 & 100 \\
LST & 318 & 264 & 716 & 824 & 775 & 68.4 & 100 & 100 \\
MCF & 220 & 478 & 1,215 & 1,049 & 980 & 50 & 100 & 100 \\
MCT & 400 & 337 & 633 & 973 & 958 & 46.9 & 100 & 100 \\
MST & 452 & 293 & 854 & 1,323 & 1302 & 80 & 100 \\
PMF & 207 & 126 & 621 & 1,425 & 1248 & 83.3 & 100 \\
PST & 497 & 423 & 851 & 1,344 & 1326 & 75.3 & 98.8 \\
\hline
\end{tabular}

\section{CONCLUSIONS AND FUTURE WORK}

\subsection{CONCLUSIONS ON ENDF/B-VIII.0 COVARIANCE TESTING}

A variety of methods are used to assess new covariance data, including comparing the energy-dependent sensitivity profiles (Section 3.1), comparing the predicted data-induced uncertainty in $k_{\text {eff }}$ to the distribution of $\mathrm{C} / \mathrm{E}$ values for different categories of critical experiments (Section 3.2), and comparing the applicability of a suite of critical experiments to a known system for which selecting experiments is unclear (Section 3.3). All of the assessments point to a general increase in the uncertainties in the nuclear data and thus an increase in the estimated nuclear data induced uncertainty in $k_{\text {eff. }}$

A number of data handling issues have been encountered in the analysis presented here, though few of them have been mentioned explicitly. The most common problem is associated with threshold reactions. Absolute uncertainties can result in very large uncertainties averaged over a group when only a small portion of the group has any cross section for the threshold reaction. Relative uncertainties of tens of thousands of percent have been noted during this work; this is an unavoidable pitfall for multigroup covariance data with real threshold reactions. This emphasizes the importance of proper handling of the data both in the processing codes (e.g., AMPX) and the analysis codes (e.g., TSUNAMI-IP) and an awareness of the potential problems.

\subsection{FOLLOW-ON WORK ON THE AUGMENTED COVARIANCE MATRIX}

In the follow-on work on augmenting the ENDF/B-VIII.0 covariance matrix, one of the guiding principles will be that small cross-correlations are conservative compared to large cross-correlations where no correlations currently exist. The conservative nature of smaller cross-correlations as opposed to larger one is independent of the sign. This is because of the interpretation of the correlation coefficient between two random variables. The correlation coefficient can be interpreted as the predictive power of one variable on another. A positive or negative correlation measures how much knowing the value of one variable can reduce the confidence interval on another variable. Therefore, a statement of no predictive power, i.e. a zero-correlation coefficient, is conservative. The goal of the following analysis will be to 
realistically estimate how much the individual nuclear data quantities vary together without over-stating the confidence of being able to predict their co-variation.

Further analysis is warranted of the including different integral benchmarks in the TSURFER GLLS calculations. The case of ${ }^{239} \mathrm{Pu}$ cross-correlations provides a convincing argument that the sign and approximate magnitude of the cross correlations is only weakly dependent on the choice of integral benchmarks. However, this idea must be studied further especially as the energy dimension will be refined from the coarse group of the current analysis to increasingly fine group treatment.

In this first augmentation of the ENDF/B-VIII.0 covariance matrix, only the fission to nu-bar cross correlations have been examined as they were judged to be the most influential missing piece of the covariance data. In further analysis, missing cross correlations for other nuclear data quantities will be estimated. Ultimately, the cross-correlations which have already been estimated based on the differential data analysis, such as fission to capture cross sections will be re-examined from the perspective of the integral experiments feedback loop.

Refining the energy dimension and examining the cross-correlations between other nuclear data quantities will seek to ensure that there is a balance between accurately stating the increased predictive power of modeling nuclear systems neutronically similar to the integral benchmarks and honestly acknowledging the lack of predictive power in nuclear systems which are different from the integral benchmarks used to improve the nuclear data.

\section{REFERENCES}

1. F. Bostelmann, A. M. Holcomb, J. B. Clarity, W. J. Marshall, V. Sobes, and B. T. Rearden, Nuclear Data Performance Assessment for Advanced Reactors, ORNL/TM-2018/1033, UTBattelle, LLC, Oak Ridge National Laboratory (2019).

2. D. A. Brown et al., "ENDF/B-VIII.0: The $8^{\text {th }}$ Major Release of the Nuclear Reaction Data Library with CIELO-project Cross Sections, New Standards and Thermal Scattering Data," Nucl. Data Sheets 148, pp. 1-142 (2018).

4. B. T. Rearden et al., "Sensitivity and Uncertainty Analysis Capabilities and Data in SCALE," Nucl. Technol. 174(2), 236-288 (2011).

5. W. J. Marshall and B. T. Rearden, "The SCALE Verified, Archived Library of Inputs and DataVALID," ANS Nuclear Criticality Safety Division Topical Meetings (NCSD2013), Wilmington, NC (2013).

6. J. M. Scaglione et al., An Approach for Validating Actinide and Fission Product Burnup Credit Criticality Safety Analyses-Criticality ( $k_{\text {eff) }}$ Predictions, NUREG/CR-7109 (ORNL/TM2011/514), prepared for the US Nuclear Regulatory Commission (NRC) by Oak Ridge National Laboratory, Oak Ridge, TN (2012).

7. W.J. Marshall et al., Technical Basis for Peak Reactivity Burnup Credit for BWR Spent Nuclear Fuel in Storage and Transportation Systems, NUREG/CR-7194 (ORNL/TM-2014/240) (2015).

8. B. T. Rearden and M. A. Jessee, Editors, SCALE Code System, ORNL/TM-2005/39, Version 6.2.2, Oak Ridge National Laboratory (2017).

9. R. Little et al., "Low-fidelity Covariance Project," Nuclear Data Sheets 109(12), pp. 2828-2833 (2008).

10. Division of Spent Fuel Storage and Transportation, Interim Staff Guidance-8, Revision 3, Burnup Credit in the Criticality Safety Analyses of PWR Spent Fuel in Transportation and Storage Casks, US Nuclear Regulatory Commission (2012).

11. W. J. Marshall, B. J. Ade, S. M. Bowman, I. C. Gauld, G. Ilas, U. Mertyurek, and G. Radulescu, Technical Basis for Peak Reactivity Burnup Credit for BWR Spent Nuclear Fuel in Storage and Transportation Systems, NUREG/CR-7194 (ORNL/TM-2014/240), prepared for the US Nuclear Regulatory Commission by Oak Ridge National Laboratory, Oak Ridge, TN (2015). 
12. I. Hill, J. Gulliford, J. B. Briggs, B. T. Rearden, and T. Ivanova, "Generation of 1800 New Sensitivity Data Files for ICSBEP Using SCALE 6.0," Trans. of the American Nuclear Society 109(1), pp. 867-869 (2013).

13. D. E. Mueller, K. R. Elam, and P. B. Fox, Evaluation of the French Haut Taux de Combustion (HTC) Critical Experiment Data, NUREG/CR-6979 (ORNL/TM-2007/083) prepared for the US Nuclear Regulatory Commission by Oak Ridge National Laboratory, Oak Ridge, TN (2008).

14. International Handbook of Reactor Physics Experiments, "Evaluation of the Initial Critical Configuration of the HTR-10 Pebble-Bed Reactor," HTR10-GCR-RESR-001, NEA/NSC/DOC(2006)1, Rev. 0, 2007.

15. International Handbook of Reactor Physics Experiments, "Evaluation of the Start-up Core Physics Tests at Japan's High Temperature Engineering Test Reactor (Fully-Loaded Core)," HTTR-GCR-RESR-001, NEA/NSC/DOC(2006)1, Rev. 1, 2010.

16. E.E. Sunny, G. Ilas, "SCALE 6 analysis of HTR-10 pebble-bed reactor for initial critical configuration", PHYSOR 2010 International Conference on Reactor Physics, Pittsburgh, PA, May 2010.

17. G. Ilas, "On SCALE Validation for PBR Analysis", PHYSOR 2010 International Conference on Reactor Physics, Pittsburgh, PA, May 2010.

18. G. Ilas et al., "Validation of SCALE for High Temperature Gas-Cooled Reactor Analysis," NUREG/CR-7107, ORNL/TM-2011/161, 2012.

19. B. R. Betzler et al. 2017. "Molten Salt Reactor and Fuel Cycle Modeling and Simulation with SCALE," Annals of Nuclear Energy 101, pp. 489-503.

20. B. R. Betzler et al. 2017. "Assessment of Neutronic and Fuel Cycle Performance of the Transatomic Power Molten Salt Reactor,” ORNL/TM-2017/475, CRADA/NFE-16-06345.

21. B. R. Betzler et al. 2017. "Two-Dimensional Neutronic and Fuel Cycle Analysis of the Transatomic Power Molten Salt Reactor," ORNL/TM-2016/742.

22. B. R. Betzler et al. 2017. "Fuel Cycle Analysis of Fast and Thermal Molten Salt Reactors," Proc. GLOBAL International Fuel Cycle Conference, Seoul, Korea, Sep. 24-29.

23. B. R. Betzler et al. 2018. "Fuel Cycle and Neutronic Performance of a Spectral Shift Molten Salt Reactor Design," Annals of Nuclear Energy 119, pp. 396-410.

24. E. E. Davidson et al. 2018. "Modeling a Fast Molten Salt Reactor with ORION," Proc. $15^{\text {th }}$ NEA Information Exchange Meeting on Actinide and Fission Product Partitioning and Transmutation, Manchester Hall, Manchester, UK.

25. L. Buiron, G. Rimpault, et al.,2017. Benchmark for Uncertainty Analysis in Modelling (UAM) for Design, Operation and Safety Analysis of SFRs, Core Definitions, Version 1.5, Rev. 10, OECD/NEA, 2017.

26. F. Bostelmann, W. Zwermann, et al., 2018. Benchmark for Uncertainty Analysis in Modeling (UAM) for Design, Operation and Safety Analysis of SFRs, Definition of Sub-Exercises, OECD/NEA, 2018. 